\title{
A NONLINEAR OBLIQUE DERIVATIVE BOUNDARY VALUE PROBLEM FOR THE HEAT EQUATION: ANALOGY WITH THE POROUS MEDIUM EQUATION
}

\author{
Luis A. CAFFARELLI ${ }^{\mathrm{a}}$, Jean-Michel ROQUEJOFFRE ${ }^{\mathrm{b}}$ \\ a Department of Mathematics, University of Texas at Austin, Austin, TX 78712-1082, USA \\ ${ }^{\mathrm{b}}$ UFR-MIG, UMR CNRS 5640, Université Paul Sabatier, 118 route de Narbonne, \\ 31062 Toulouse Cedex, France
}

Received 8 September 2000, revised 1 February 2001

\begin{abstract}
The problem under investigation is the heat equation in the upper half-plane, to which the diffusion in the longitudinal direction has been suppressed, and augmented with a nonlinear oblique derivative condition. This paper proves global existence and qualitative properties to the Cauchy problem for this model, furthering the study [18] of the self-similar solutions. The qualitative behaviour of the solutions exhibits a strong analogy with the porous medium equation: propagation with compact support and finite speed, free boundary relation and time-asymptotic convergence to self-similar solutions.
\end{abstract}

(C) 2002 L'Association Publications de l'Institut Henri Poincaré. Published by Elsevier B.V. All rights reserved

RÉSUMÉ. - Le problème étudié concerne l'équation de la chaleur dans le demi-plan supérieur, sans diffusion longitudinale, avec une condition à la limite non linéaire. On démontre l'existence globale d'une solution au problème de Cauchy, ainsi que diverses propriétés qualitatives. En particulier, les solutions présentent une forte analogie avec celles de l'équation des milieux poreux, comme : la propagation à vitesse finie, la relation de frontière libre et la convergence en temps grand vers des solutions auto-similaires.

(C) 2002 L'Association Publications de l'Institut Henri Poincaré. Published by Elsevier B.V. All rights reserved

\section{Introduction}

Let $\mathbb{R}_{+}^{2}$ be the upper half-plane

$$
\mathbb{R}_{+}^{2}=\left\{(z, x) \in \mathbb{R} \times \mathbb{R}_{+}\right\}
$$

and set

$$
\Gamma=\{t>0, z \in \mathbb{R}, x=0\} .
$$

E-mail addresses: caffarel@math.utexas.edu (L.A.Caffarelli), roque@mip.ups_tlse.fr (J.-M. Roquejoffre). 
We are interested in the problem

$$
\begin{aligned}
u_{t}-u_{x x} & =0, \quad(t, z, x) \in \mathbb{R}_{+} \times \mathbb{R}_{+}^{2}, \\
u_{x} & =u u_{z}, \quad(t, z, x) \in \Gamma,
\end{aligned}
$$

with the condition at $\pm \infty$

$$
u(t,-\infty, x)=1, \quad u(t,+\infty, x)=0 .
$$

This equation occurs in the modelling of a plasma opening switch. Consider a plasma injected in the half-plane $\mathbb{R}_{+}^{2}$ through an anode placed on the axis $\{x=0\}$. It can be described as a fluid in the setting of electron magnetohydrodynamics. Let $u$ be the magnetic field, propagating in the plasma. If certain conditions are fulfilled the field is a solution of the system (1.2) after a rescaling. The key here is the boundary condition on the anode. This electrode is a perfect conductor so the electric field is perpendicular to it, which gives, with Ohm's law, the nonlinear condition $u_{x}=u u_{z}$ on $\{x=0\}$. For the modelling, see [12,16].

The goal of this paper is to study existence and qualitative properties, furthering [17] - the problem with full diffusion - and [18] - self-similar solutions for (1.2), (1.3). It was already noticed in $[12,18]$ that the self-similar problem had some resemblance with the porous medium equation

$$
u_{t}=\left(\frac{u^{3}}{3}\right)_{z z} \quad(t>0, z>0)
$$

posed on $\Gamma$. In particular, the support of the self-similar solution, restricted to the portion of the boundary $\{z>0, x=0\}$ is compact.

We would like to extend this analogy to the full Cauchy problem. To back this impression, let us do the following heuristics: setting $\phi(t, z)=u(t, z, 0)$ we write

$$
\begin{aligned}
\phi_{t} & =u_{x x}(t, z, 0) \\
& "="\left(u u_{z}\right)_{x}(t, z, 0) \\
& =\left(u u_{z x}(t, z, 0)+\phi \phi_{z}^{2}=\phi\left(\phi \phi_{z}\right)_{z}+\phi \phi_{z}^{2}=\left(\frac{\phi^{3}}{3}\right)_{z z} .\right.
\end{aligned}
$$

The second line of this series of equalities is utterly wrong, but we will prove there is some truth in these heuristics. The porous medium equation, posed on the half line, with fixed condition at the boundary, has among others - the following features:

- Finite speed propagation and compactly supported solutions,

- existence and global stability of self-similar solutions [3,4],

- linear behaviour of the function $p=u^{2}$ near the free boundary and free boundary relation [2].

Although Problem (1.2) is essentially a 1D problem - this statement will be reinforced in the next section, when we derive an integral equation for $u$ - the methods developed in [2] - to get the existence and the smoothness of the free boundary - do not seem to 
apply here. They indeed heavily rely on the well-known Aronson-Bénilan estimate [1]:

$$
\Delta p \geqslant-\frac{C}{t} .
$$

The spirit of this study will therefore be more in the spirit of the multi-D papers $[8,9]$. Indeed, the multi-D porous medium equation

$$
u_{t}=\Delta\left(\frac{u^{3}}{3}\right)
$$

besides having compactly supported solutions and finite speed of propagation, enjoys free boundary smoothness properties. The main tools are rescalings through a double homogeneity property of the pressure, Harnack inequalities and an iteration argument. They also rely on the Aronson-Bénilan estimate, but we will see that this difficulty may be bypassed in the present context.

All in all, the main theorem of this paper is

THEOREM 1.1. - Assume the initial datum $u_{0}$ to satisfy

- We have $u_{0} \equiv 1$ on $\left\{(z, x) \in \mathbb{R}_{-} \times \mathbb{R}_{+}\right\}$,

- there exists $\zeta_{0}>0$ such that $u_{0}(z, x)>0$ iff $z<\zeta_{0}$,

- $u_{0}$ is smooth on its positivity set - except perhaps, at the line $\{x=0\}$, where it may be discontinuous,

- $\partial_{z} u_{0}, \partial_{x} u_{0} \leqslant 0 ; \partial_{x x} u_{0}>0$ on $] 0, \zeta_{0}\left[\times \mathbb{R}_{+}\right.$,

- there exists a smooth function $f(z, x)$, locally bounded away from 0 in $\left[0, \zeta_{0}\right] \times \mathbb{R}_{+}$, such that

$$
u_{0}(z, x)=f(z, x) \sqrt{\zeta_{0}-z} .
$$

Then Problem (1.2), (1.3) has a unique global classical solution. Moreover there is a $C^{1, \alpha}$ function $\zeta(t)$ such that

- for all $(t, z, x) \in \mathbb{R}_{+} \times \mathbb{R}_{+}^{2}, u(t, z, x)>0$ if and only if $z<\zeta(t)$,

- the function $u$ is smooth on its positivity set-except, perhaps, on the line $\{x=0\}$. Moreover the 'pressure' function

$$
p=u^{2}
$$

is smooth up to the boundary of its positivity set.

The proof of this result will take most of the paper. A by-product of this theorem is a strong comparison principle that will allow us to prove the next

THEOREM 1.2. - Assume the initial datum to satisfy the assumptions of Theorem 1.1. There exists $z_{0} \in \mathbb{R}$ such that

$$
\lim _{t \rightarrow+\infty} u(t, z \sqrt{t}, x \sqrt{t})=\phi\left(z+z_{0}, x\right) .
$$

Finally, we will complete the analogy with the porous medium equation by deriving a free boundary relation. 
THEOREM 1.3 (Free boundary relation). - Assume the initial datum to satisfy the assumptions of Theorem 1.1. The following relation is valid for all $t>0$ :

$$
p_{t}(t, \zeta(t), 0)=\frac{1}{4 \pi} p_{z}^{2}(t, \zeta(t), 0) .
$$

As a consequence the speed of the interface is given by

$$
\dot{\zeta}(t)=-\frac{1}{4 \pi} p_{z}(t, \zeta(t), 0) .
$$

The plan of this paper is as follows. Because it seem hard to construct solutions to (1.2), (1.3) in a direct fashion, we approximate the problem by just replacing the condition $u=0$ at $+\infty$ by $u=\varepsilon$. If we believe there is some element of truth in the porous medium heuristics, then we should be facing a strictly parabolic nonlinear diffusion problem - or at least, something quite equivalent - and we will indeed see that this is what happens. A cornerstone of the section will be a comparison principle for the classical solutions, that will remain valid for pointwise limits of classical solutions. The outcome of the section will be a global viscosity solution to (1.2), (1.3).

We will pause in Section 3 to examine some special solutions, namely: travelling waves and self-similar solutions. The latter were studied at length in [18], but we will take this occasion to prove that they are viscosity solutions - a rather necessary property if we wish to compare them to other viscosity solutions.

The study of the free boundary really starts in Section 4 . We will basically prove in this section the results corresponding to [8]: the free boundary is Lipschitz and nondegenerate. The spirit of the proofs will more or less be the same as in [8], but we will have to devote an extra effort to prove a class of Harnack inequalities suited to our problem.

Section 5 will adapt the iteration technique of [9] to the present context and yield Theorem 1.1. Finally, Theorems 1.2 and 1.3 will be proved in Section 6.

\section{Smooth approximations}

As shown by the heuristics of the introduction, the nonlinear oblique derivative boundary condition acts as a nonlinear effective diffusion at the boundary. Instead of asking $u$ to go to 0 as $z \rightarrow+\infty$, we therefore investigate the solutions $u_{l_{-}, l_{+}}$of the problem

$$
\left\{\begin{array}{rlrl}
u_{t}-u_{x x} & =0, & & (t, z, x) \in \mathbb{R}_{+} \times \mathbb{R}_{+}^{2}, \\
u_{x}=u u_{z} & (t>0, z \in \mathbb{R}, x=0)
\end{array}\right.
$$

with the conditions at $\pm \infty$

$$
u(t,-\infty, x)=l_{-}, \quad u(t,+\infty, x)=l_{+} ; \quad 0<l_{+} \leqslant l_{-} .
$$

For commodity, the subscript $l_{-}, l_{+}$will be deleted, except in the last paragraph of this section.

The 'pressure' $p=u^{2}$ satisfies the equation 


$$
\left\{\begin{aligned}
& p_{t}-p_{x x}=-\frac{p_{x}^{2}}{4 p},(t, z, x) \in \mathbb{R}_{+} \times \mathbb{R}_{+}^{2}, \\
& p_{x}=\sqrt{p} p_{z} \quad(t>0, z \in \mathbb{R}, x=0) .
\end{aligned}\right.
$$

The organisation of the section is the following: first, we derive the homogeneity and comparison principles for the positive solutions of (2.1); then we prove a global existence result to the Cauchy problem for (2.1), (2.2). We end the section by defining the solutions to Problem (1.2).

Notations. In the whole paper, we will define the following boxes:

$$
\begin{aligned}
& B_{h}^{-}\left(t_{0}, z_{0}\right)=\left\{-h<t-t_{0}<0,-h<z-z_{0}<h\right\}, \\
& B_{h}^{+}\left(t_{0}, z_{0}\right)=\left\{0<t-t_{0}<h,-h<z-z_{0}<h\right\}, \\
& B_{h}\left(t_{0}, z_{0}\right)=B_{h}^{+}\left(t_{0}, z_{0}\right) \cup B_{h}^{-}\left(t_{0}, z_{0}\right),
\end{aligned}
$$

\subsection{Homogeneity, comparison principle}

We begin with homogeneity considerations, and notice that Eq. (2.1) has the same two-parameter family of homogeneities as the porous medium equation. Namely:

PROPOSITION 2.1. - Let $u$ be a solution of (2.1). Then, for all nonzero $b$ and $c$, the function $\frac{c}{b} u\left(\frac{t}{b^{2}}, \frac{z}{c}, \frac{x}{b}\right)$ is also a solution to (2.1). Similarly, if $p$ is a solution of (2.3), then $\frac{c^{2}}{b^{2}} p\left(\frac{t}{b^{2}}, \frac{z}{c}, \frac{x}{b}\right)$ is also a solution to $(2.3)$.

The proof is obvious.

The basis of the whole theory is that two smooth solutions of (2.1), (2.2) which compare at the time $t=0$ will also compare at all later time. We note here that, because we expect an interface to the solution of the initial problem (1.2), we cannot talk yet about smooth solutions, even for the pressure $p$, that we only expect to be Lipschitz.

PROPOSITION 2.2. - Let $u_{10} \leqslant u_{20}$ be two positive smooth Cauchy data for (2.1), such that $\partial_{z} u_{0 i} \leqslant 0$. Assume they generate two global classical solutions $u_{1}$ and $u_{2}$. Then we have $\partial_{z} u_{i} \leqslant 0$, and $u_{1} \leqslant u_{2}$.

Proof. - Assume first $\partial_{z} u_{i} \leqslant 0$ and set $v=u_{1}-u_{2}-\delta$; the boundary condition for $v$ is

$$
v_{x}=\left(\left(u_{1}+u_{2}\right) v\right)_{z}+\delta\left(u_{1}+u_{2}\right)_{z} .
$$

We may, for instance, multiply the equation for $v$ by $v^{+}$and integrate on $\mathbb{R}_{+}^{2}$. Because we have $\left(u_{1}+u_{2}\right)_{z} \leqslant 0$ we obtain

$$
\begin{aligned}
& \frac{1}{2} \frac{\mathrm{d}}{\mathrm{d} t} \int_{\mathbb{R}_{+}^{2}}\left(v^{+}\right)^{2} \mathrm{~d} z \mathrm{~d} x+\int_{\mathbb{R}_{+}^{2}}\left(\left(v^{+}\right)_{x}\right)^{2} \mathrm{~d} z \mathrm{~d} x \\
& \quad+\frac{1}{4} \int_{-\infty}^{+\infty}\left(u_{1}+u_{2}\right)_{z}(t, z, 0)\left(v^{+}\right)^{2}(t, z, 0) \mathrm{d} z \leqslant 0 .
\end{aligned}
$$


Because $u_{1}$ and $u_{2}$ are smooth, the boundary term is controlled by the two volume integrals of $\left(v^{+}\right)^{2}$ and $\left(\left(v^{+}\right)_{x}\right)^{2}$, and we conclude with Gronwall's lemma. Then we send $\delta$ to 0 .

To prove that $\partial_{z} u_{i} \leqslant 0$, we set this time $v=\partial_{z} u_{i}$ and we note that $v^{+}=0$ at $t=0$. The boundary condition is this time

$$
v_{x}=u_{i} v_{z}+v^{2}
$$

multiplying by $v^{+}$, integrating by parts and using the smoothness of $v$ yields $v^{+} \equiv 0$.

We point out that the assumption $\partial_{z} u_{0} \leqslant 0$ is most certainly unnecessary. It simplifies, however, the proof of this proposition, which is important enough to deserve a selfcontained proof. On the other hand, Proposition 2.2 is not quite sufficient to handle all the comparisons that we are going to make in the sequel of the paper. Therefore we generalize it to the

PROPOSITION 2.3. - Let $u_{1}$ and $u_{2}$ be respectively a weak sub-solution and a weak super-solution for (2.1), with initial data $u_{0 i}$, such that

- $\partial_{z} u_{i} \leqslant 0$ in the distributional sense,

- there exist two constants $\left(a_{i}\right)_{i \in\{1,2\}}$ such that $u_{i}$ is smooth on $\left\{t \geqslant 0, z \leqslant a_{i}, x \geqslant 0\right\}$ and $\left\{t \geqslant 0, z \geqslant a_{i}, x \geqslant 0\right\}$,

- $u_{01} \leqslant u_{02}$.

Then we have $u_{1} \leqslant u_{2}$.

Proof. - One should first note that, under the assumptions of the proposition, the function $z \mapsto u_{2}(t, z, 0)$ is continuous for all $t>0$. If it were not so, the bounded term $u_{x}$ would have to be less than - in the distribution sense $-u u_{z}$, which carries a Dirac measure; this is a contradiction. Then we may argue just as in Proposition 2.2.

The reason why we need this proposition is that we shall have to handle self-similar solutions, which behave exactly in the fashion described by Proposition 2.3. Another extension of Proposition 2.2 concerns the problem in the quarter plane

$$
\begin{aligned}
& u_{t}-u_{x x}=0, \quad(t, z, x) \in \mathbb{R}_{+} \times \mathbb{R}_{+} \times \mathbb{R}_{+}, \\
& u_{x}=u u_{z}, \quad(t, z) \in \mathbb{R}_{+} \times \mathbb{R}_{+}, x=0,
\end{aligned}
$$

with the Dirichlet condition

$$
u(t, 0,0)=\alpha(t),
$$

where $\alpha(t)$ is a given $C^{1}$ function.

PROPOSITION 2.4. - Let $u_{1}$ and $u_{2}$ be respectively a weak sub-solution and a weak super-solution for (2.6), with initial data $u_{0 i}$, such that

- $\partial_{z} u_{i} \leqslant 0$ in the distributional sense,

- there exist two nonnegative constants $\left(a_{i}\right)_{i \in\{1,2\}}$ such that $u_{i}$ is smooth on $\{t \geqslant$ $\left.0,0 \leqslant z \leqslant a_{i}, x \geqslant 0\right\}$ and $\left\{t \geqslant 0, z \geqslant a_{i}, x \geqslant 0\right\}$,

- $u_{1}(t, 0,0) \leqslant \alpha(t) \leqslant u_{2}(t, 0,0)$,

- $u_{01} \leqslant u_{02}$.

Then we have $u_{1} \leqslant u_{2}$. 
This will also be needed in the course of the paper.

A first consequence of Proposition 2.2 is

Proposition 2.5. - The Cauchy problem for (2.1), (2.2) has at most one smooth solution, provided that the initial datum is $z$-decreasing.

Another consequence is

Proposition 2.6. - Assume that the Cauchy datum $u_{0}$ satisfies the assumptions of Proposition 2.2, and moreover satisfies $\partial_{x} u_{0} \leqslant 0, \partial_{x x}^{2} u_{0} \geqslant 0$. Assume it generates a classical solution $u$; then we have $\partial_{x} u \leqslant 0, \partial_{x x}^{2} u \geqslant 0$.

The next consequence of the proposition is a hyperbolic inequation verified by the classical solutions of (2.1).

Proposition 2.7. - Assume $u_{0}$ to satisfy $\partial_{x} u_{0} \leqslant 0, \partial_{z} u_{0} \leqslant 0$, and to generate $a$ classical solution u to (2.1), (2.2). Then

$$
\forall(t, z, x) \in \mathbb{R}_{+} \times \mathbb{R}_{+}^{2}, \quad 2 t u_{t}+x u_{x}+z u_{z} \leqslant 0 .
$$

Proof. - For all $\varepsilon>0$ we have

$$
u_{0}\left(\frac{z}{1+\varepsilon}, \frac{x}{1+\varepsilon}\right) \geqslant u_{0}(z, x) .
$$

In Proposition 2.5 we take $c=b=1+\varepsilon$; Proposition 2.5 implies that the only solution of (2.1) with datum $u_{0}\left(\frac{z}{1+\varepsilon}, \frac{x}{1+\varepsilon}\right)$ is $u\left(\frac{t}{(1+\varepsilon)^{2}}, \frac{z}{1+\varepsilon}, \frac{x}{1+\varepsilon}\right)$. From Proposition 2.2 and (2.8) we have

$$
u\left(\frac{t}{(1+\varepsilon)^{2}}, \frac{z}{1+\varepsilon}, \frac{x}{1+\varepsilon}\right) \geqslant u(t, z, x) .
$$

Then we expand the above inequality with respect to $\varepsilon$, divide by $\varepsilon$ and send $\varepsilon$ to 0 .

COROLlary 2.1. - Assume the assumptions of Proposition 2.6 to be satisfied. Then $u_{z}$ controls $u_{t}$ on $\{t>0, z>0, x \geqslant 0\}$.

To see this, we first set $x=0$, which implies the result at the boundary. The corollary is true in the whole domain because both $u_{t}$ and $u_{z}$ satisfy the $1 \mathrm{D}$ heat equation.

All in all, we see that a lower estimate for $u_{z}$ will imply a bound for $u$ in Lipschitz norm. Moreover, this will imply that the level sets of $u$ will have finite, controlled speeds.

\subsection{The Cauchy problem for (2.1), (2.2)}

The main result of this section is the following.

Theorem 2.1. - Choose $\alpha \in] 0, \frac{1}{2}\left[\right.$. Let $u_{0}$ be a $C^{2, \alpha}$ datum satisfying (2.2), and

$$
\partial_{x} u_{0}, \partial_{z} u_{0} \leqslant 0, \quad \partial_{x x}^{2} u_{0} \geqslant 0 .
$$

Then (2.1), (2.2) has a unique global classical solution $u$, satisfying inequalities (2.10). Moreover, $u$ is $C^{\infty}$ if $u_{0}$ is $C^{\infty}$. 
Of course, the important matter is what happens at the boundary $\{x=0\}$, since $u$ satisfies the $1 \mathrm{D}$ heat equation inside the domain. Let $I_{1 / 2}$ denote the Abel time-integrator of order $1 / 2$. For any function $f(t)$ locally bounded on $\mathbb{R}_{+}^{*}$ it is defined as - see [11]:

$$
I_{1 / 2} f=\frac{1}{\sqrt{\pi}} \int_{0}^{t} \frac{f(\tau)}{\sqrt{t-\tau}} \mathrm{d} \tau .
$$

Let $\partial_{1 / 2}$ denote the time half-derivative

$$
\partial_{1 / 2} f(t)=\frac{1}{\sqrt{\pi}} \frac{\mathrm{d}}{\mathrm{d} t} \int_{0}^{t} \frac{f(s)}{\sqrt{t-s}} \mathrm{~d} s=\frac{1}{\sqrt{\pi}} \int_{0}^{t} \frac{\dot{f}(s)}{\sqrt{t-s}} \mathrm{~d} s
$$

the last equality being valid only if $f(0)=0$. We have [11]

$$
\begin{aligned}
& I_{1 / 2} I_{1 / 2} f(t)=\int_{0}^{t} f(s) \mathrm{d} s, \\
& \partial_{1 / 2} \partial_{1 / 2} f(t)=\dot{f}(t), \quad \partial_{1 / 2} I_{1 / 2} f(t)=f(t) .
\end{aligned}
$$

Let us set $\phi(t, z)=u(t, z, 0)$, the function $\phi$ satisfies the integral equation

$$
\phi(t, z)=\left(e^{t \partial_{x x}^{2}} u_{0}\right)(z, 0)-I_{1 / 2}\left(\phi \phi_{z}\right) .
$$

For integral operators of Abel type and their properties, see, for instance, [11,19]. Global existence results related to Theorem 2.1 are proved in recent works of Clément, Gripenberg and Londen [10,13]. They consider equations of type $u=I_{\alpha}\left(f(u)_{z}\right)+u_{0}$, where $I_{\alpha}$ is the Abel integrator of order $\left.\alpha \in\right] 0,1[$; the function $f$ is smooth and satisfies $f^{\prime} \geqslant \delta>0$. They use sectorial operator and analytic semigroup techniques, which do not seem to localize easily. Because we do need local smoothness results in the rest of the paper, we think that it is useful to present a complete independent existence theory.

The proof of Theorem 2.1 comprises two steps: first, existence of a maximal solution; second, uniform estimates proving the existence of the solution for all times. The first step is relatively standard. The second step goes as follows: formulation (2.14) may be iterated to yield

$$
\phi(t, z)=\left(e^{t \partial_{x x}^{2}} u_{0}\right)(z, 0)-I_{1 / 2}\left(\phi\left(\phi \partial_{z}\left(\left(e^{t \partial_{x x}^{2}} u_{0}\right)(z, 0)-I_{1 / 2}\left(\phi \phi_{z}\right)\right)_{z}\right)\right) .
$$

Given Eq. (2.13), this formulation resembles much to a nonlinear parabolic equation of the form

$$
\phi_{t}=\left(\phi^{2} \phi_{z}\right)_{z}+\text { lower order terms }
$$

which brings us back to the porous medium equation - but, this time, with a solution that is bounded away from 0 . The standard way to prove regularity for such an eqution is

(i) to prove a gradient estimate, so that the term $\phi^{2} \phi_{z z}$ appears as a term of the form $a(t, z) \phi_{z z}$ with $a$ Hölder;

(ii) apply Schauder estimates combined to a suitable frozen coefficients technique [15]. 
Lemma 2.1. - There is a $t_{\max }>0$ such that Problem (2.1), (2.2) has a unique classical solution of $\left[0, t_{\max }[\right.$.

For this, we linearize Eq. (2.14) around the initial value $\phi_{0}$ and write an approximate diffusion equation for $\phi$. What makes Lemma 2.1 hold is the

Lemma 2.2. - Consider $f(t, z) \in C^{2+\alpha, 1+\frac{\alpha}{2}}\left(\mathbb{R}_{+} \times \mathbb{R}\right)$ such that $f(0, z)=0$ for all $z$ and two functions $a(z), b(z) \in C^{2, \alpha}(\mathbb{R})$ such that $a \geqslant a_{0}>0$. There is a unique function $\phi(t, z)$ solution of

$$
\phi=I_{1 / 2}\left(a(z) \phi_{z}+b(z) \phi\right)+f(t, z) .
$$

Moreover there is a constant $C\left(a_{0},\|a\|_{2, \alpha}\right)$ such that

$$
\|\phi\|_{2+\alpha, 1+\frac{\alpha}{2}} \leqslant C\|f\|_{2+\alpha, 1+\frac{\alpha}{2}} .
$$

For the proof of this lemma, the following preliminary is needed.

Lemma 2.3. - Consider $f(t, z) \in C^{2+\alpha, 1+\frac{\alpha}{2}}\left(\mathbb{R}_{+} \times \mathbb{R}\right)$ such that $f(0, z)=0$. Then we have

$$
\left\|\partial_{1 / 2} f_{z}\right\|_{\alpha, \frac{\alpha}{2}}+\left\|\partial_{1 / 2} f\right\|_{\alpha, \frac{\alpha}{2}} \leqslant C\|f\|_{2+\alpha, 1+\frac{\alpha}{2}} .
$$

Proof. - The nontrivial part concerns of course the Hölder norm of $\partial_{1 / 2} f_{z}$. We break the difference $\partial_{1 / 2} f_{z}(t, z)-\partial_{1 / 2} f_{z}\left(t^{\prime}, z^{\prime}\right)$ into the sum of three integrals:

$$
\begin{aligned}
& I_{1}=\int_{0}^{t} \frac{f_{z t}(s, z)-f_{z t}\left(s, z^{\prime}\right)}{\sqrt{t-s}} \mathrm{~d} s, \\
& I_{2}=\int_{0}^{t} f_{z t}\left(s, z^{\prime}\right)\left((t-s)^{-1 / 2}-\left(t^{\prime}-s\right)^{-1 / 2}\right) \mathrm{d} s, \\
& I_{3}=\int_{t}^{t^{\prime}} \frac{f_{z t}\left(s, z^{\prime}\right)}{\sqrt{t^{\prime}-s}} \mathrm{~d} s .
\end{aligned}
$$

Only $I_{1}$ will be evaluated, the two others being treated similarly. We integrate by parts and obtain

$$
I_{1}=\int_{0}^{t} \frac{f_{z}(s, z)-f_{z}(t, z)-f_{z}\left(s, z^{\prime}\right)+f_{z}\left(t, z^{\prime}\right)}{(t-s)^{3 / 2}} \mathrm{~d} s .
$$

Then the integral is broken into two regions:

$$
I_{1}=\int_{0}^{t-\left(z-z^{\prime}\right)^{2}}+\int_{t-\left(z-z^{\prime}\right)^{2}}^{t}:=I_{11}+I_{12}
$$

To treat $I_{12}$, we use the fact that, for all $(s, t, z) \in \mathbb{R}_{+} \times \mathbb{R}_{+} \times \mathbb{R}$, we have

$$
\left|f_{z}(t, z)-f_{z}(s, z)\right| \leqslant\|f\|_{2+\alpha, 1+\frac{\alpha}{2}}|t-s|^{\frac{1+\alpha}{2}}
$$


and a similar property for $\left|f_{z}\left(t, z^{\prime}\right)-f_{z}\left(s, z^{\prime}\right)\right|$. All in all, we control $I_{12}$ by $\left|z-z^{\prime}\right|^{\alpha}$. As for $I_{11}$, we use

$$
\Delta\left(s, z, z^{\prime}\right):=\left|f_{z}\left(s, z^{\prime}\right)-f_{z}(s, z)-f_{z z}(s, z)\left(z-z^{\prime}\right)\right| \leqslant\|f\|_{2+\alpha, 1+\frac{\alpha}{2}}\left|z-z^{\prime}\right|^{1+\alpha}
$$

and we have

$$
\begin{aligned}
I_{11} & \leqslant \int_{0}^{t-\left(z-z^{\prime}\right)^{2}} \frac{\left|f_{z z}(t, z)-f_{z z}(s, z)\right|\left|z^{\prime}-z\right|+\Delta\left(s, z, z^{\prime}\right)+\Delta\left(t, z, z^{\prime}\right)}{(t-s)^{3 / 2}} \mathrm{~d} s \\
& \leqslant C\|f\|_{2+\alpha, 1+\frac{\alpha}{2}} \int_{0}^{t-\left(z-z^{\prime}\right)^{2}} \frac{(t-s)^{\alpha / 2}\left|z^{\prime}-z\right|+\left|z^{\prime}-z\right|^{1+\alpha}}{(t-s)^{3 / 2}} \mathrm{~d} s
\end{aligned}
$$

and we end up with a control of $I_{11}$ by $\left|z-z^{\prime}\right|^{\alpha}$.

Proof of Lemma 2.2. - The idea is to write a linear diffusion equation for $\phi$ and to use the classical Schauder estimates. Assuming $\phi$ to exist, we see that it has to satisfy

$$
\begin{aligned}
\phi & =I_{1 / 2}\left(a(z) \phi_{z}+b(z) \phi\right)+f \\
& =I_{1 / 2}\left(a(z)\left(I_{1 / 2}\left(a(z) \phi_{z}+b(z) \phi\right)+f\right)_{z}+b(z) I_{1 / 2}\left(a(z) \phi_{z}+b(z) \phi+f\right)\right)+f
\end{aligned}
$$

hence the parabolic equation

$$
\phi_{t}=a\left(a \phi_{z}\right)_{z}+a(b \phi)_{z}+a b \phi_{z}+b^{2} \phi+a \partial_{1 / 2} f_{z}+b \partial_{1 / 2} f+f_{t} .
$$

From Lemma 2.3, $\partial_{1 / 2} f$ and $\partial_{1 / 2} f_{z}$ are controlled in $C^{\alpha, \alpha / 2}$ by the $C^{2+\alpha, 1+\alpha / 2}$-norm of $f$. The coefficients $a, a_{z}$ and $b$ being more than $C^{\alpha}$, the classical Schauder estimates apply.

Proof of Lemma 2.1. - If such a solution $\phi$ exists, the function $\varphi$ obtained by setting

$$
\begin{aligned}
f_{0}(t, z) & =e^{t \partial_{x x} u_{0}}(z, 0), \\
f_{1}(t, z) & =f_{0}+I_{1 / 2}\left(f_{0} \partial_{z} f_{0}\right), \\
\phi & =f_{1}+\varphi
\end{aligned}
$$

verifies the equation

$$
\varphi=I_{1 / 2}\left(f_{1} \partial_{z} f_{1}+f_{1} \varphi_{z}+\partial_{z} f_{1} \varphi+\varphi \varphi_{z}\right) .
$$

A fixed point argument is built up as follows: for a given $T>0$, and a given element $\psi(t, z) \in C^{2+\alpha, 1+\alpha / 2}([0, T] \times \mathbb{R})$, we define $\mathcal{T} \psi$ as the solution $\varphi$ of

$$
\begin{aligned}
\varphi= & I_{1 / 2}\left(\partial_{z} f_{1}(0, z) \varphi+f_{1}(0, z) \varphi_{z}\right) \\
& +I_{1 / 2}\left(\partial_{z}\left(f_{1}-f_{1}(0, z)\right) \psi+\left(f_{1}-f_{1}(0, z)\right) \psi_{z}+\psi \psi_{z}\right) .
\end{aligned}
$$

If $T>0$ is small enough, the $C^{2+\alpha, 1+\alpha / 2}$ norm of the linear terms terms in $\psi$ may be estimated by $T^{\alpha}\|\psi\|_{2+\alpha, 1+\alpha / 2}$. Lemma 2.2 then ensures that $\mathcal{T}$ is a contraction on a suitably small ball of $C^{2+\alpha, 1+\alpha / 2}([0, T] \times \mathbb{R})$. 
To prove that we have $t_{\max }=+\infty$, we need $a$ priori estimates. They are broken into two steps: first, a Lipschitz bound; then the final $C^{2+\alpha, 1+\alpha / 2}$ bound.

LEMMA 2.4. - Assume the assumptions of Theorem 2.1 to hold. There is a constant $C\left(l_{-}, l_{+}\right)$such that any classical solution of Problem (2.1), (2.2) with initial datum $u_{0}$ satisfies $\left\|u_{t}\right\|_{\infty}+\left\|u_{x}\right\|_{\infty}+\left\|u_{z}\right\|_{\infty} \leqslant C / \sqrt{t}$.

Proof. - From Corollary 2.1, it is sufficient to give a lower bound for $u_{z}$. Clearly, we have $l_{-} \leqslant u \leqslant l_{+}$. Then we set $v(t, z, x)=u_{x}(t, z, x)$ and write

$$
\begin{aligned}
v_{x}(t, z, 0) & =u_{x x}(t, z, 0)=u_{t}(t, z, 0) \\
& \leqslant-C u_{z}(t, z, 0) \quad \text { from Proposition } 2.7 \\
& \leqslant-\frac{C}{l_{-}} v(t, z, 0) .
\end{aligned}
$$

Then we still denote by $u_{x}$ the even extension of $u_{x}$ to $\mathbb{R}_{+} \times \mathbb{R} \times \mathbb{R}_{-}$. It satisfies

$$
v_{t}-v_{x x} \geqslant-\frac{C}{l_{-}} v \delta_{x=0},
$$

which classically implies

$$
v(t, z, 0) \geqslant e^{t \partial_{x x} v_{0}}-\frac{C}{l_{-}} I_{1 / 2} v .
$$

The first term of the right-hand side is estimated from below by $-C l_{+} t^{-1 / 2}$, and we infer from the generalized Gronwall lemma [14, Ch. 7] that $v(t, z, 0) \geqslant-C\left(l_{-}, l_{+}\right) t^{-1 / 2}$. Then we use the boundary condition $u_{z}=v / u$ to conclude that

$$
u_{z}(t, z, 0) \geqslant-\frac{C\left(l_{-}, l_{+}\right)}{l_{-}} t^{-1 / 2} .
$$

Lemma 2.4 may be localized, and its local version is stated without proof in the following proposition.

Proposition 2.8. - Assume $u>0$ to satisfy Problem (2.1) in $B_{1}\left(t_{0}, z_{0}\right) \times \mathbb{R}_{+}$. Also assume $u_{t}, u_{z}, u_{x} \leqslant 0$. Then $\left|u_{x}(0,0,0)\right|+\left|u_{z}(0,0,0)\right|+\left|u_{t}(0,0,0)\right|$ is controlled by a constant only depending on $\max _{B_{1}\left(t_{0}, z_{0}, 0\right)} u$ and $\min _{B_{1}\left(t_{0}, z_{0}, 0\right)} u$.

The $C^{2+\alpha, 1+\alpha / 2}$ estimates are now provided in their local versions. In contrast with the Lipschitz estimates, there does not seem to be an easy way to derive them globally. In order to do this, we scale the equation so that the boundary condition reads $u_{x}=$ $(1+\mathrm{o}(1)) u_{z}$ and apply Lemma 2.2. This absorbs the o(1) $u_{z}$.

PROPOSITION 2.9. - Under the assumptions of Proposition 2.8, and for all $r>0$, there exists a constant $C$ depending on $r, \max _{B_{r}\left(t_{0}, z_{0}\right)} u, \min _{B_{r}\left(t_{0}, z_{0}\right)} u$ and

$$
\sup _{x \geqslant 0}\left\|u_{0}(., x)\right\|_{C^{0, \alpha}\left(\left[z_{0}-r, z_{0}+r\right]\right)}
$$

such that 


$$
\|u\|_{C^{2+\alpha, 1+\alpha / 2\left(B_{r}\left(t_{0}, z_{0}\right)\right.}} \leqslant C .
$$

Remark 2.1. - There is in this problem not a full regularizing effect as there would be in a diffusion equation; there is instead a memory effect preventing a parabolic equation type bootstrap. The term preventing the bootstrap is $\partial_{\eta} e^{t \partial_{\xi \xi}^{2}} u_{0}$, which remains even after localization, and for which there is no smoothing effect in $z$. For instance, assume that $u_{0}(z, x)$ has a discontinuity line $\{z=0\}$. The maximum regularity that we may recover with such a term is Lipschitz regularity in $z$. However, the derivative discontinuity will remain located at the line $\{z=0\}$. This is why the $z$-derivative of the self-similar solution is discontinuous at the point $(z=0, x=0)$, see [18].

To prove the proposition, we will need a lemma quite similar in spirit to Lemma 2.3.

Lemma 2.5. - Choose $T>0$. Let $a(t, z)$ be a Lipschitz function in $t$ and $z$ and $b(t, z)$ belong to $L^{p}([0, T] \times \mathbb{R})$, with $p>2$. Let the function $\psi(t, z)$ be defined as

$$
\psi=I_{1 / 2}\left(a I_{1 / 2} b\right) .
$$

(i) The function $\psi_{t}$ belongs to $L^{p}([0, T] \times \mathbb{R})$; moreover we have

$$
\left\|\psi_{t}\right\|_{L^{p}([0, T] \times \mathbb{R})} \leqslant C\|a\|_{\text {Lip }}\|b\|_{L^{p}([0, T] \times \mathbb{R})} .
$$

(ii) Assume $b$ to be in $C^{\alpha, \alpha / 2}\left(\mathbb{R}_{+} \times \mathbb{R}\right)$. Then $\psi_{t}$ belongs to $C^{\alpha, \alpha / 2}\left(\mathbb{R}_{+} \times \mathbb{R}\right)$ and we have

$$
\begin{aligned}
\left|\psi_{t}(t, z)-\psi_{t}\left(t^{\prime}, z^{\prime}\right)\right| \leqslant & C\left(1+\|a\|_{\text {Lip }}\right)\|b\|_{\infty} \sqrt{\left|z-z^{\prime}\right|+\left|t-t^{\prime}\right|} \\
& +C\left(1+\|a\|_{\infty}\right)\|b\|_{\alpha, \alpha / 2}\left(\left|z-z^{\prime}\right|^{2}+\left|t-t^{\prime}\right|\right)^{\alpha / 2}
\end{aligned}
$$

Proof. - First, we claim that

$$
\begin{aligned}
\psi_{t} & =a b-\frac{1}{2 \sqrt{\pi}} \int_{0}^{t} \frac{a(s, .)-a(t, .)}{(t-s)^{3 / 2}} I_{1 / 2} b(s, .) \mathrm{d} s \\
& :=a b-\frac{1}{2 \sqrt{\pi}} \psi_{1} .
\end{aligned}
$$

To prove formula (2.28), one only has to decompose $\psi$ under the form

$$
\psi=a \int_{0}^{t} b \mathrm{~d} s+\frac{1}{\sqrt{\pi}} \int_{0}^{t} \frac{a(s, .)-a(t, .)}{\sqrt{t-s}} I_{1 / 2} b(s, .) \mathrm{d} s
$$

and differentiate with respect to $t$, assuming that $a$ is $C^{1}$ - because the intermediate computations involve $a_{t}$. Then one concludes by a density argument.

Inequality (2.26) is now obvious. To prove inequality (2.27), we assume without loss of generality that $t \leqslant t^{\prime}$ and we break the difference $\psi_{1}(t, z)-\psi_{1}\left(t^{\prime}, z^{\prime}\right)$ into the sum $\frac{1}{\sqrt{\pi}}\left(I_{1}+I_{2}+I_{3}\right)$, where

$$
I_{1}=\int_{t}^{t^{\prime}} \frac{a\left(s, z^{\prime}\right)-a\left(t^{\prime}, z^{\prime}\right)}{\left(t^{\prime}-s\right)^{3 / 2}} I_{1 / 2} b\left(s, z^{\prime}\right) \mathrm{d} s,
$$




$$
\begin{aligned}
& I_{2}=\int_{0}^{t}\left(\frac{a\left(s, z^{\prime}\right)-a\left(t^{\prime}, z^{\prime}\right)}{\left(t^{\prime}-s\right)^{3 / 2}}-\frac{a(s, z)-a(t, z)}{\left(t^{\prime}-s\right)^{3 / 2}}\right) I_{1 / 2} b\left(s, z^{\prime}\right) \mathrm{d} s, \\
& I_{3}=\int_{0}^{t} \frac{a(s, z)-a(t, z)}{(t-s)^{3 / 2}}\left(I_{1 / 2} b\left(s, z^{\prime}\right)-I_{1 / 2} b(s, z)\right) \mathrm{d} s .
\end{aligned}
$$

To bound $I_{1}$, we use the fact that $a$ is Lipschitz and $b$ bounded, and we easily obtain a control of $I_{1}$ by $\|b\|_{\infty}\|a\|_{\text {Lip }} \sqrt{t^{\prime}-t}$.

To bound $I_{2}$, we break it under the form

$$
I_{2}=\int_{0}^{t-\left|z-z^{\prime}\right|}+\int_{t-\left|z-z^{\prime}\right|}^{t}:=I_{21}+I_{22}
$$

We have

$$
\begin{aligned}
\left|I_{21}\right|= & C\|b\|_{\infty}\left(\int _ { 0 } ^ { t - | z - z ^ { \prime } | } \left(\frac{\left|a\left(s, z^{\prime}\right)-a(s, z)\right|}{\left(t^{\prime}-s\right)^{3 / 2}}+\frac{\left|a\left(t^{\prime}, z^{\prime}\right)-a(t, z)\right|}{\left(t^{\prime}-s\right)^{3 / 2}}+\cdots\right.\right. \\
& \left.\left.+|a(t, z)-a(s, z)|\left(\frac{1}{(t-s)^{3 / 2}}-\frac{1}{\left(t^{\prime}-s\right)^{3 / 2}}\right)\right) \mathrm{d} s\right) \\
\leqslant & C\|b\|_{\infty}\|a\|_{\text {Lip }}\left(\sqrt{t^{\prime}-t}+2 \sqrt{\left|z^{\prime}-z\right|}+\int_{0}^{t}\left(\frac{1}{\sqrt{t-s}}-\frac{t-s}{\left(t^{\prime}-s\right)^{3 / 2}}\right) \mathrm{d} s\right) \\
\leqslant & 2 C\|b\|_{\infty}\|a\|_{\text {Lip }}\left(\sqrt{t^{\prime}-t}+\sqrt{\left|z^{\prime}-z\right|}\right) .
\end{aligned}
$$

According to the above calculation, the only term that we still have to estimate in $I_{22}$ is

$$
\tilde{I}_{22}:=\int_{t-\left|z-z^{\prime}\right|}^{t} \frac{\left|a\left(s, z^{\prime}\right)-a(s, z)+a(t, z)-a\left(t^{\prime}, z^{\prime}\right)\right|}{\left(t^{\prime}-s\right)^{3 / 2}} \mathrm{~d} s,
$$

taking into account that we still bound $I_{1 / 2} b$ by $\|b\|_{\infty}$. We write

$$
\begin{aligned}
\tilde{I}_{22} & \leqslant \int_{t-\left|z-z^{\prime}\right|}^{t} \frac{\left|a\left(s, z^{\prime}\right)-a\left(t^{\prime}, z^{\prime}\right)\right|}{\left(t^{\prime}-s\right)^{3 / 2}} \mathrm{~d} s+\int_{t-\left|z-z^{\prime}\right|}^{t} \frac{\left|a(s, z)-a\left(t, z^{\prime}\right)\right|}{\left(t^{\prime}-s\right)^{3 / 2}} \mathrm{~d} s \\
& \leqslant\|a\|_{\text {Lip }}\left(\int_{t-\left|z-z^{\prime}\right|}^{t} \frac{\mathrm{d} s}{\sqrt{t^{\prime}-s}}+\int_{t-\left|z-z^{\prime}\right|}^{t} \frac{t-s}{\sqrt{t^{\prime}-s}} \mathrm{~d} s\right) \\
& \leqslant 2\|a\|_{\text {Lip }}\left(\sqrt{\left|z-z^{\prime}\right|}+\sqrt{t^{\prime}-t}\right) .
\end{aligned}
$$

All in all, we have, for some constant $C>0$ : 


$$
\left|I_{2}\right| \leqslant C\|b\|_{\infty}\|a\|_{\operatorname{Lip}}\left(\sqrt{t^{\prime}-t}+\sqrt{\left|z^{\prime}-z\right|}\right) .
$$

The term $I_{3}$ is much easier to bound; one may see only by inspection that

$$
\left|I_{3}\right| \leqslant C\|a\|_{\text {Lip }}\|b\|_{\alpha, \alpha / 2}\left|z-z^{\prime}\right|^{\alpha}
$$

Gathering all the information, we prove the lemma.

Remark 2.2. - Lemma 2.5 would still be true if, instead of assuming $a$ to be Lipschitz, we had assumed it to be $\mu$-Hölder in $t$ and $z$, with $\left.\mu \in] \frac{1}{2}, 1\right]$.

Proof of Proposition 2.9. - First, we set $\beta:=u\left(t_{0}, z_{0}, 0\right)$ and

$$
h u(\tau, \eta, \xi):=u\left(t_{0}+h^{2}(\tau-1), z_{0}+h \eta, h \xi\right)-\beta,
$$

where $h<1$ is a small parameter such that $t_{0}-3 h>0$. Then, let $\gamma(\tau, \eta)$ be a nonnegative mollifier vanishing outside the ball $B_{2}(1,0)$ and equal to 1 in $B_{1}(1,0)$ and let us set $v(\tau, \eta)=(\gamma u)(\tau, \eta)$. The equation satisfied by $v$ reads

$$
\left\{\begin{aligned}
v_{\tau}-v_{\xi \xi} & =\gamma_{\tau} u, & & (\tau, \eta, \xi) \in \mathbb{R}_{+} \times \mathbb{R}_{+}^{2}, \\
v_{\xi} & =(\beta+h u)\left(v_{\eta}-\gamma_{\eta} u\right) & & (\tau>0, \eta \in \mathbb{R}, \xi=0) .
\end{aligned}\right.
$$

Setting

$$
w(\tau, \eta)=\int_{-1}^{\tau}\left(e^{(\tau-\sigma) \partial_{\xi \xi}} \gamma_{\tau} u\right)_{\xi=0} \mathrm{~d} \sigma
$$

we write

$$
v=I_{1 / 2}\left((\beta+h u)\left(v_{\eta}-\gamma_{\eta} u\right)\right)+w .
$$

Our task is now to write a diffusion equation for $v$, with small nonlinear parts. To do so, we iterate Eq. (2.33), and we end up with an expression of the form

$$
v_{\tau}=\beta^{2} v_{\eta \eta}+\partial_{\tau}\left(J_{1}+J_{2}+J_{3}\right)
$$

where

$$
\begin{aligned}
& J_{1}=I_{1 / 2}\left((\beta+h u) I_{1 / 2}(\beta+h u) v_{\eta \eta}\right)-\beta^{2} \int_{-1}^{\tau} v_{\eta \eta} \mathrm{d} s, \\
& J_{2}=I_{1 / 2}\left((\beta+h u) I_{1 / 2}(\beta+h u)_{\eta} v_{\eta}\right)-I_{1 / 2}\left((\beta+h u) I_{1 / 2}\left(\gamma_{\eta} u\right)_{\eta}\right), \\
& J_{3}=w+I_{1 / 2}\left((\beta+h u) w_{\eta}\right) .
\end{aligned}
$$

To prove our proposition, we have to control the $C^{\alpha, \alpha / 2}$ norm of $\partial_{\tau}\left(J_{1}+J_{2}\right)$.

According to Lemma 2.5(i), we may bound the $L^{p}$ norm of $\partial_{\tau}\left(J_{1}+J_{2}\right)$ by $C_{p}(1+$ $\left.h\left\|v_{\eta \eta}\right\|_{L^{p}([-1,1] \times \mathbb{R})}\right)$. Indeed, let us first have a look at $J_{1}$; we set $J_{1}=J_{11}+J_{12}$, with 


$$
\begin{aligned}
& J_{11}=h I_{1 / 2}\left(u I_{1 / 2}(\beta+h u) v_{\eta \eta}\right)+h^{2} I_{1 / 2}\left(u I_{1 / 2}\left(u v_{\eta \eta}\right)\right) \\
& J_{12}=h \beta \int_{-1}^{\tau} u v_{\eta \eta} \mathrm{d} \sigma .
\end{aligned}
$$

We then estimate $\partial_{\tau} J_{11}$ by application of Lemma 2.5(i), by remembering that:

- the $\eta$-Lipschitz norm of $u$ is preserved under rescaling,

- the $\tau$-Lipschitz norm of $u$ becomes multiplied by $h$.

Then we notice that $\partial_{\tau} J_{12}=h \beta u v_{\eta} \eta$. The remaining terms in $J_{1}$ involve lower order terms in $u$ and $v$. The term $J_{2}$ involves lower order terms in $v$, that are under control. Finally, $\left\|\partial_{\tau} J_{3}\right\|_{L^{p}}$ is controlled by $\sup _{-1 \leqslant s \leqslant 1}\left\|u_{\eta}(s, ., .)\right\|_{L^{\infty}\left(\mathbb{R}_{+}^{2}\right)}$, which is itself controlled by

$$
\sup _{-1 \leqslant s \leqslant 1}\left\|u_{\eta}(s, ., 0)\right\|_{L^{\infty}(\mathbb{R})}+\sup _{-1 \leqslant s \leqslant 1}\left\|\partial_{\eta} e^{s \partial_{\xi \xi} u_{0}}\right\|_{L^{\infty}\left(\mathbb{R}_{+}^{2}\right)}
$$

by the maximum principle. Hence this quantity is under control, provided the quantity given by Eq. (2.23) is also controlled.

Then we apply the parabolic $L^{p}$ estimates, that tell us

$$
\left\|\left(v_{\tau}, v_{\eta}, v_{\eta \eta}\right)\right\|_{L^{p}([-1,1] \times \mathbb{R})} \leqslant C_{p}\left(1+h\left\|v_{\eta \eta}\right\|_{L^{p}([-1,1] \times \mathbb{R})}\right) .
$$

For $h$ small enough, we have the desired control. Now, undoing the scaling, we cover the ball $B_{r}\left(t_{0}, z_{0}\right)$ with a finite number of balls of radius $h$ and apply the just found estimate in each of them.

A first consequence of the above considerations is that, because $p$ can be chosen large enough, we have a control on $u_{z}$ in $C^{\alpha, \alpha / 2}$ norm. Let us now redo the scaling (2.31) and scan back the terms $\partial_{\tau} J_{1}$ and $\partial_{\tau} J_{2}$, given by (2.34).

Application of Lemma 2.5(ii) with $a:=u, b=(\beta+h u) v_{\eta \eta}$ yields the estimate

$$
\left\|\partial_{\tau} J_{11}\right\|_{\alpha, \alpha / 2} \leqslant C\left(1+h\left\|v_{\eta \eta}\right\|_{\alpha, \alpha / 2}\right) \text {. }
$$

Because now the function $u_{z}$ is bounded in $C^{\alpha, \alpha / 2}$, the remaining terms are also bounded in $C^{\alpha, \alpha / 2}$. Lemma 2.5(ii) applies once again.

Then we apply the parabolic Schauder estimates, that tell us

$$
\left\|\left(v_{\tau}, v_{\eta}, v_{\eta \eta}\right)\right\|_{\alpha, \alpha / 2} \leqslant C\left(1+h\left\|v_{\eta \eta}\right\|_{L^{p}([-1,1] \times \mathbb{R})}\right) .
$$

This ends the proof of the proposition.

Proof of Theorem 2.1. - On the one hand, we have a local existence theorem with a maximum life time $t_{\max }$ for the solution $u$. On the other hand, Proposition 2.9 tells us that the $C^{2+\alpha, 1+\alpha / 2}$ norm of $u$ is estimated up to $t_{\max }$. This means $t_{\max }=+\infty$.

The fact that $u$ is $C^{\infty}$ if $u_{0}$ is $C^{\infty}$ can now be inferred from a standard botstrap procedure. 


\subsection{Construction of a solution to the original problem}

Coming back to the notations of the beginning of this section, we call $u^{\varepsilon}$ the unique solution of the Cauchy problem for (2.1), with $l_{-}=1+\varepsilon$ and $l_{+}=\varepsilon$. Thus we build a nonincreasing sequence of smooth functions, which therefore converges pointwise and in $L_{\text {loc }}^{1}\left(\mathbb{R}_{+}^{2}\right)$ to some function $u(t, z, x)$. Let us list the obvious properties of $u$ :

- we have $0 \leqslant u \leqslant 1$,

- the function $u$ is nondecreasing in $t$ and nonincreasing in $z$ and $x$;

- the function $u$ is $C^{\infty}$ in $x$ for $x>0$ - not up to the boundary - and convex in $x$. These facts have several consequences. The first one is the

Proposition 2.10. - The function $u$ is a weak solution to (1.2).

Proof. - Let $\varphi(t, z, x)$ be a smooth compactly supported test function; for all $\varepsilon>0$ we have

$$
\int_{\mathbb{R}_{+} \times \mathbb{R}_{+}^{2}}\left(-\varphi_{t}-\varphi_{x x}\right) u^{\varepsilon}-\int_{\Gamma}\left(\frac{u^{\varepsilon 2}}{2} \varphi_{z}-u^{\varepsilon} \varphi_{x}\right) \mathrm{d} s \mathrm{~d} z=\int_{\mathbb{R}_{+}^{2}} u_{0}^{\varepsilon} \varphi .
$$

Because of items 1 and 2, the family $\left(u^{\varepsilon}\right)_{\varepsilon}$ is bounded in $B V\left(\mathbb{R}_{+}^{2}\right)$; let us denote by $\gamma$ the trace at the boundary $\Gamma=\{t>0, x=0, z \in \mathbb{R}\}$. Because $\gamma$ is continuous from $B V\left(\mathbb{R}_{+}^{2}\right)$ to $L^{1}(\Gamma)$, we may pass to the limit $\varepsilon \rightarrow 0$ in Eq. (2.36).

The limits (1.3) at $\pm \infty$ hold because it is possible to trap $u$ between two similarity solutions that are known to exist - see next section.

Proposition 2.10 is rather anecdotical: first, because we will prove that $u$ is a classical solution on its positivity set, with a free boundary relation. Second, because such a formulation does not allow us to prove a comparison principle, which is the cornerstone of the theory. This is why we are not going to dwell any longer on the notion of weak solution.

Viscosity solutions to the porous media equation have already been defined in Caffarelli and Vazquez [7] and have been proved to have comparison properties. We could, if we wished so, give intrinsic definitions to our equation, but we will not do so. The definition of viscosity solution that we will adopt is the following:

DEFINITION 2.1. - Let $u_{0}$ be a smooth initial datum satisfying:

- $\partial_{z} u_{0} \leqslant 0, \partial_{x} u_{0} \leqslant 0, \partial_{x x} u_{0} \geqslant 0$.

- $u_{0}(-\infty, x)=1$, uniformly in $x$, and there exists $z_{0}>0$ such that $u_{0}(z, 0)=0$ for $z \geqslant z_{0}$.

A viscosity solution to (1.2) with initial datum $u_{0}$ is the pointwise limit, as $\varepsilon \rightarrow 0$, of the family $\left(u^{\varepsilon}\right)_{\varepsilon}$ of classical solutions of $(2.1)$, with initial data $u^{\varepsilon}(0)$ such that $u^{\varepsilon}(0)$ has limit $\varepsilon$ as $z \rightarrow+\infty, 1+\mathrm{O}(\varepsilon)$ as $z \rightarrow-\infty$, and such that $\left(u^{\varepsilon}(0)\right)_{\varepsilon}$ converges uniformly to $u_{0}$ as $\varepsilon \rightarrow 0$.

First, we notice that the limit does not depend on the approximation of $u_{0}$. Indeed, $\left(u^{\varepsilon}(0)\right)_{\varepsilon}$ and $\left(\tilde{u}^{\varepsilon}(0)\right)_{\varepsilon}$ be two approximating sequences of $u_{0}$; for all $\delta>0$ we may find a sequence $\left(\varepsilon_{n}\right)_{n}$ such that $u^{\varepsilon_{n}}(0) \leqslant \tilde{u}^{\varepsilon_{n}}(0)+\delta$; hence if $u$ and $\tilde{u}$ are the corresponding limits we have $u \leqslant \tilde{u}+\delta$. The definition therefore implies immediately the following result, which is the conclusion of this section. 
THEOREM 2.2. - The Cauchy problem (1.2) has a unique viscosity solution. Moreover, two viscosity solutions of Problem (1.2) that compare initially will compare at all later times.

\section{Special solutions}

\subsection{Self-similar solutions}

A self-similar solution of Problem (2.1) is a solution of the form $u(t, z, x)=$ $\phi\left(\frac{z}{\sqrt{t}}, \frac{x}{\sqrt{t}}\right)$. Such a solution $\phi(z, x)$ satisfies

$$
\begin{aligned}
-\phi_{x x}-\frac{1}{2}\left(z \phi_{z}+x \phi_{x}\right) & =0 & & \left(\mathbb{R}_{+}^{2}\right), \\
\phi_{x} & =\phi \phi_{z} & & (x=0) .
\end{aligned}
$$

The problem is supplemented by the condition

$$
\phi(-\infty, x)=M>0, \quad \phi(+\infty, x)=0 .
$$

We have the

THEOREM 3.1. - Problem (3.1), (3.2) has a unique viscosity solution $\phi_{M}$. Moreover there exists $z_{M}>0$ such that $\phi_{M}$ verifies

$$
\begin{gathered}
\phi_{M} \equiv M \quad \text { on } \mathbb{R}_{-}^{*} \times \mathbb{R}_{+}, \\
\phi_{M} \equiv 0 \quad \text { on }\left[z_{0},+\infty\left[\times \mathbb{R}_{+},\right.\right. \\
\phi_{M} \text { is discontinuous along the axis } z=0, x>0, \\
\phi_{M} \in C^{\infty}(] 0, z_{M}\left[\times \mathbb{R}_{+}\right), \\
\left.\phi_{M}(., 0) \text { is continuous and Lipschitz on }\right]-\infty, z_{M}[.
\end{gathered}
$$

Proof. - The existence of a solution $\phi_{M}$ to (3.1), (3.2) with properties (3.4)-(3.7) is asserted by Theorem 1.1 of [18]. To see that it is a viscosity solution, let us construct $\varepsilon$-approximations $\phi^{\varepsilon}$ of $\phi_{M}$; we drop the subscript ${ }_{M}$. We notice that $\phi^{\varepsilon}$ is still discontinuous across the $x$-line, but we may restrict ourselves to the construction of $\phi^{\varepsilon}$ on $\mathbb{R}_{+}^{2}$; the condition at point $(0,0)$ is $\phi^{\varepsilon}=1+\varepsilon$. We extend $\phi^{\varepsilon}$ by $1+\varepsilon$ for negative $z$. The condition at $+\infty$ becomes $\phi(+\infty, x)=\varepsilon$, uniformly in $x$. A super-solution $\bar{\phi}^{\varepsilon}$ is given by the solution $\psi_{1+\varepsilon}$ of the linear problem of the next paragraph. A subsolution is given by $\phi^{\varepsilon}=\phi+\varepsilon$, and we have $\bar{\phi}^{\varepsilon} \geqslant \underline{\phi}^{\varepsilon}$. Hence there is a solution $\phi^{\varepsilon}$, which is smooth from Theorem 1.1 of [18].

Finally, we notice that all $\varepsilon$-approximations of Problem (3.1), (3.2) are nonincreasing in $z$; hence all viscosity solutions of this problem have this property. Hence a viscosity solution of (3.1), (3.2) falls in the category of entropy soutions of (3.1), (3.2) as defined in [18]; therefore Theorem 1.2 of [18] implies uniqueness and, as a consequence, the uniform convergence of $\phi^{\varepsilon}$ to $\phi$. 
Let us call $\zeta_{M}(t)$ the interface of the self-similar solution $\phi_{M}\left(\frac{z}{\sqrt{t}}, \frac{x}{\sqrt{t}}\right)$. From the homogeneity considerations of Section 2.1 , we have

$$
\phi_{M}(z)=M \phi_{1}\left(\frac{z}{M}, 1\right), \quad z_{M}=M z_{1}, \quad \zeta_{M}(t)=M z_{1} \sqrt{t} .
$$

The last information that we need about the self-similar solutions is their behaviours near $z=z_{M}$.

Proposition 3.1. - There exists $k>0$ such that $\phi_{1}(z, 0) \sim k \sqrt{z_{1}-z}$ as $z \rightarrow z_{1}$.

Proof. - Set $\varphi(z)=\phi_{1}(z, 0)$. We start from the Cauchy formula

$$
\varphi\left(\frac{z}{\sqrt{t}}\right)=\left.e^{t \partial_{x x}^{2}}\left(\lim _{t \rightarrow 0} \varphi\left(\frac{z}{\sqrt{t}}, \frac{x}{\sqrt{t}}\right)\right)\right|_{x=0}-\int_{0}^{t} \varphi\left(\frac{z}{\sqrt{s}}\right) \varphi^{\prime}\left(\frac{z}{\sqrt{s}}\right) \frac{\mathrm{d} s}{\sqrt{t-s}}
$$

from which we obtain, after sending $t \rightarrow+\infty$ and setting $\eta=\frac{z}{\sqrt{t}}$ :

$$
\varphi(\eta)=-\frac{1}{\sqrt{\pi}} \int_{0}^{1} \varphi\left(\frac{\eta}{\sqrt{\sigma}}\right) \varphi^{\prime}\left(\frac{z \eta}{\sqrt{\sigma}}\right) \frac{\mathrm{d} \sigma}{\sqrt{1-\sigma}} .
$$

Taking into account that $\varphi(\eta)=0$ if $\eta \geqslant z_{1}$, we choose $\eta<z_{1}$; then set $\eta=z_{1}-t$ and $\varphi(t):=\varphi\left(z_{1}-t\right)$. Eq. (3.9) becomes

$$
\varphi(t)=\frac{2\left(z_{1}-t\right)}{\sqrt{\pi}} \int_{0}^{t} \frac{\varphi(s) \varphi^{\prime}(s)}{\sqrt{(t-s)\left(2 z_{1}-t-s\right)}\left(z_{1}-s\right)} \mathrm{d} s .
$$

Let us now recall that we are working with small $t>0$; hence we may set, for all small $t>0,0 \leqslant s<t$ :

$$
\frac{1}{\sqrt{(t-s)\left(2 z_{1}-t-s\right)}\left(z_{1}-s\right)}=\frac{1}{\sqrt{2}\left(z_{1}-t\right)^{3 / 2}}+(t-s) h(s, t),
$$

where $h$ is $C^{\infty}$ in a neighbourhood of $(0,0)$. Let us set $\Pi(t)=\varphi^{2}(t)$; we have

$$
\frac{\sqrt{\Pi(t)}}{z_{1}-t}=\frac{1}{\sqrt{2}\left(z_{1}-t\right)^{3 / 2}} \partial_{1 / 2} \Pi+I_{1 / 2}(\Pi h)-\frac{1}{\sqrt{\pi}} \int_{0}^{t} \Pi(s) h_{s}(s, t) \sqrt{t-s} \mathrm{~d} s .
$$

The idea is that $\Pi$ almost solves the equation $\Pi=$ Constant $I_{1 / 2} \sqrt{\Pi}$, to which explicit solutions are easily computed. Comparison with these solutions will give the right behaviour of $\Pi$ at $t=0$, hence the proposition.

Set

$$
\mathcal{L} \Pi=\frac{1}{\sqrt{2}}\left(\left(z_{1}-t\right)^{3 / 2} I_{1 / 2}(\Pi h)\right)-\left(\sqrt{2}\left(z_{1}-t\right)^{3 / 2} \int_{0}^{t}\left(\Pi h_{s} \sqrt{t-s}\right)\right) ;
$$


then, in the vicinity of $t=0, \Pi$ solves the equation

$$
\left(I+I_{1 / 2} \mathcal{L}\right) \Pi=I_{1 / 2}\left(\sqrt{2\left(z_{1}-t\right) \Pi}\right) .
$$

For every $\varepsilon>0$, there exists a solution $\underline{\Pi}^{\varepsilon}(t)$ to the integral equation

$$
\underline{\Pi}=(1-\varepsilon) I_{1 / 2}\left(\sqrt{2 z_{1} \Pi}\right)
$$

given by

$$
\underline{\Pi}^{\varepsilon}(t)=\frac{2(1-\varepsilon)^{2} z_{1}}{\pi} t .
$$

Let us define $t_{\varepsilon}>0$ such that we have, for all $t \leqslant t_{\varepsilon}$ :

$$
\sqrt{2\left(z_{1}-t\right) \Pi}-\mathcal{L} \Pi(t) \geqslant(1-\varepsilon) \sqrt{2 z_{1}} \Pi(t) .
$$

This is indeed possible, since $\Pi(t)$ is an increasing function and because of the inequality $\mathcal{L} \Pi(t) \leqslant C t \max _{0 \leqslant s \leqslant t} \Pi(s)$. Now, as in [5], we lift $\Pi$ and $\underline{\Pi}^{\varepsilon}$ on the interval $\left[0, t_{\varepsilon}\right]$. We have $\Pi(t)=Q(t, 0)$ and $\underline{\Pi}^{\varepsilon}(t)=\underline{Q}^{\varepsilon}(t, 0)$, the functions $Q(t, z)$ and $\underline{Q}^{\varepsilon}(t, z)$ being initially 0 and satisfying the singular heat equation

$$
\begin{aligned}
\partial_{t} Q-\partial_{z z} Q & =2\left(\sqrt{2\left(z_{1}-t\right) Q}-\mathcal{L} Q\right) \delta_{z=0} \quad(t>0, \quad z \in \mathbb{R}), \\
\partial_{t} \underline{Q}^{\varepsilon}-\partial_{z z} \underline{Q}^{\varepsilon} & =2(1-\varepsilon) \sqrt{2 z_{1}} \underline{Q}^{\varepsilon} \delta_{z=0} \quad(t>0, \quad z \in \mathbb{R}) .
\end{aligned}
$$

We now claim that, for every $\mu>0$ small enough, we have $Q(t+\mu, z) \geqslant \underline{Q}^{\varepsilon}(t, z)$. Indeed, setting $R(t, z)=Q(t+\mu, z)-\underline{Q}^{\varepsilon}(t, z)$ we have, due to (3.13):

$$
\begin{aligned}
\partial_{t} R-\partial_{z z} R & \geqslant 2 \frac{(1-\varepsilon) \sqrt{2 z_{1}} R}{\sqrt{Q(t+\mu, 0)}+\sqrt{\underline{Q}^{\varepsilon}(t, 0)}} \delta_{z=0} \quad(t>0, z \in \mathbb{R}), \\
R(0, z) \geqslant 0 . &
\end{aligned}
$$

Because we have $\phi_{1}(z, 0)>0$ for $z<z_{1}$ we have $Q(t+\mu, 0) \geqslant Q(\mu, 0)>0$. Hence the denominator in the right-hand side of the equation is nonsingular, and the maximum principle applies to yield $r(t) \geqslant 0$. Sending $\mu$ to 0 yields $\Pi(t) \geqslant \underline{\Pi}^{\varepsilon}(t)$ on $\left[0, t_{\varepsilon}\right]$ and we may obtain, by the same method:

$$
\Pi(t) \leqslant \bar{\Pi}^{\varepsilon}(t):=\frac{2(1+\varepsilon)^{2} z_{1}}{\pi} t .
$$

This ends the proof of the proposition.

\subsection{Self-similar solutions of the linear problem}

Let $b>0$ be a given real number. We are interested here in the self-similar solutions of the auxiliary linear problem

$$
\begin{aligned}
u_{t}-u_{x x} & =0, \quad(t, z, x) \in \mathbb{R}_{+} \times \mathbb{R}_{+}^{2}, \\
u_{x} & =u_{z}, \quad(t, z, x) \in \Gamma,
\end{aligned}
$$


with the condition at $\pm \infty$

$$
u(t,-\infty, x)=1, \quad u(t,+\infty, x)=0 .
$$

These solutions $u(t, z, x)=\psi_{b}\left(\frac{z}{\sqrt{t}}, \frac{z}{\sqrt{t}}\right)$ satisfy

$$
\begin{aligned}
-\partial_{x x} \psi_{b}-\frac{1}{2}\left(z \partial_{z}+x \partial_{x}\right) \psi_{b} & =0 & & \left(\mathbb{R}_{+}^{2}\right), \\
\partial_{x} \psi_{b} & =b \partial_{z} \psi_{b} \quad & & (x=0)
\end{aligned}
$$

with the condition at $\pm \infty$

$$
\phi(-\infty, x)=M>0, \quad \phi(+\infty, x)=0 .
$$

The property we are interested in, and that will be useful when we prove Harnack inequalities, is the positivity of the function $\psi$. It will indeed act as a barrier function.

Proposition 3.2. - Problem (3.18), (3.19) has a unique solution $\psi_{b}$, satisfying $\psi_{b}>0, \partial_{z} \psi_{b}<0$ in $\mathbb{R}_{+}^{2}$.

Proof. - We drop the subscript ${ }_{b}$. The existence of a unique solution to (3.18), (3.19), satisfying $\partial_{z} \psi \leqslant 0$, is standard. For instance, we may think of $\psi(z, x)$ as $u_{1-H}(1, z, x)$, where $u_{1-H}$ is the solution of (3.16), (3.17), with initial datum $u_{0}(z, x)=1-H(z)$, $H$ is the Heaviside function. Strictly speaking, we have not defined yet solutions with discontinuous initial data, but we may easily interpret them as the limit of $\varepsilon$-regularizations of $1-H$. The so obtained function $\psi$ is $z$ and $x$-nonincreasing.

The function $\psi(., 0)$ has a nontrivial positivity set in $\mathbb{R}_{+}$; otherwise we would have $\psi=1-H$, and integration of (3.18), (3.19) over $\mathbb{R}_{+}^{2}$ would reveal a contradiction. Assume the existence of $z_{0}$ such that $\psi\left(z_{0}, 0\right)=0$; we may think $z_{0}$ to be the smallest $z$ satisfying $\psi(0, z)=0$. Arguing as in Proposition 3.1, we find that the function $\varphi(t)=\psi\left(z_{0}-t\right), t>0$, solves the equation

$$
\left(I+I_{1 / 2} \mathcal{L}\right) \varphi=I_{1 / 2}\left(\sqrt{2\left(z_{0}-t\right)} \varphi\right) .
$$

The notations are the same as in Proposition 3.22, up to the fact that $z_{1}$ has been replaced by $z_{0}$.

This time, the equation is linear in $\varphi$. Using the inequality $\mathcal{L} \varphi(t) \leqslant C t \varphi(t)$ and the generalized Gronwall lemma, we infer $\varphi(t)=0$ for $t>0$. This is a contradiction.

\subsection{Travelling waves}

Travelling wave solutions to the problem have the form $u(t, z, x)=l(z-V t, x)$. What really matters to us is their behaviours at $x=0$; let us therefore investigate under which conditions we have solutions of the form $l(z, 0)=k \sqrt{z^{-}}$. Let us write down the Cauchy formula (2.16) for $0<z<V t$; we obtain

$$
u(t, z, 0)=I_{1 / 2}\left(u u_{z}\right)(t, z, 0)=\frac{k^{2}}{2 \sqrt{\pi}} \int_{z / V}^{t} \frac{\mathrm{d} s}{\sqrt{t-s}}=\frac{k^{2}}{2 \sqrt{\pi}} \sqrt{\left(t-\frac{z}{V}\right)^{+}} .
$$


This imposes $k=2 \sqrt{\pi V}$, and we end up with a family of travelling waves $l_{V}(z, x)$ with

$$
l_{V}(z, 0)=\sqrt{4 \pi V z^{-}} .
$$

Recall that $l_{V}(z, x)$ satisfies the problem

$$
\begin{gathered}
-l_{x x}-V l_{z}=0, \quad(z, x) \in \mathbb{R}_{+}^{2}, \\
l_{x}=l l_{z}, \quad z \in \mathbb{R} .
\end{gathered}
$$

Proposition 3.3. - The function $l_{V}$ is the unique viscosity solution of (3.22).

Proof. - We first have to show that $l$ can be approximated from above by a sequence of classical solutions $\left(l^{\varepsilon}\right)_{\varepsilon}$ of (3.22) such that $l^{\varepsilon}(+\infty, z)=\varepsilon$. To see this we first remark that we may limit ourselves to the boundary problem in a quarter plane $(z, x) \in \mathbb{R}_{+} \times \mathbb{R}_{+}$, with the conditions

$$
l^{\varepsilon}(0,0)=1, \quad l^{\varepsilon}(+\infty, x)=\varepsilon,
$$

the last condition being uniform with respect to $x$. Indeed, assume that (3.22), (3.23) to be solved; then we extend the constructed solution - that we now call $l$ - by noticing that an integral equation for $\lambda(z):=l(z, 0)$ is given by

$$
\lambda=\left.e^{-z \partial_{x x}} l(0, .)\right|_{x=0}+\frac{1}{2 V} \partial_{1 / 2} \lambda^{2},
$$

the integrator being understood with respect to the negative $z$ variable. Eq. (3.24) may be inverted as

$$
\lambda^{2}=2 V I_{1 / 2}\left(\lambda-\left.e^{-z \partial_{x} x} l(0, .)\right|_{x=0}\right),
$$

an equation that may be solved globally in $\lambda^{2}$ by remembering that $\lambda$ has to be $\geqslant \varepsilon$.

Hence everything boils down to the problem in the quarter plane. Consider the solution $m$ of

$$
\begin{array}{rlrl}
-m_{x x}-V m_{z} & =0, & & (z, x) \in \mathbb{R}_{+}^{2}, \\
m_{x} & =m_{z}, \quad z \in \mathbb{R}, \\
m(0,0) & =1, & & m(+\infty, x)=0 .
\end{array}
$$

Given a wave solution $l_{V}$ such that $l_{V}(., 0)=1$, we have $\underline{l}^{\varepsilon}:=l_{V}+\varepsilon \leqslant \bar{l}^{\varepsilon}:=m+\varepsilon$. This is proved by multiplication of the equation for $\underline{l}^{\varepsilon}-\bar{l}^{\varepsilon}$ by $H\left(\underline{l}^{\varepsilon}-\bar{l}^{\varepsilon}\right)$ and integration by parts over the quarter plane. This yields

$$
\int_{0}^{+\infty}\left(\underline{l}^{\varepsilon}-\bar{l}^{\varepsilon}\right)(0, x) \mathrm{d} x \leqslant 0 .
$$

Hence a maximum point may only occur in the interior of the quarter plane or at the boundary away from 0 . Both are impossible: indeed, we have

$$
\left(V \partial_{z}-\partial_{x x}\right)\left(\underline{l}^{\varepsilon}-\bar{l}^{\varepsilon}\right)=0,
$$


an equation that is backward parabolic in $z$ and $x$; hence the strong maximum principle and Hopf lemma apply.

Then, we see that $\underline{l}^{\varepsilon}$ (resp. $\bar{l}^{\varepsilon}$ ) is a sub- (resp. super-) solution to our problem. Hence there is a solution $l_{V}^{\varepsilon}$ tending to $\varepsilon$ uniformly in $x$ as $z \rightarrow+\infty$, which is between $\underline{l}^{\varepsilon}$ and $\bar{l}^{\varepsilon}$. Hence $l_{V}^{\varepsilon} \geqslant \varepsilon$; hence it can be proved to be smooth, see Section 3 of [18].

Uniqueness can be proved as above, by remembering that:

(i) a viscosity solution is a weak solution, nonincreasing in $z$;

(ii) due to its definition, a viscosity solution is smooth on its positivity set.

This implies the convergence of $\left(l^{\varepsilon}\right)_{\varepsilon}$ to $l$.

\section{The free boundary and $p$ are Lipschitz and nondegenerate}

We now have the basic tools that will be needed throughout the paper, namely: existence and uniqueness of a solution having a comparison principle, and special solutions that we will be able to compare to that solution.

We initiate in this section the study of the free boundary, and all the considerations will concern the pressure $p=u^{2}$. Indeed, it can obviously be seen with Section 3 that $u$ is most unlikely to be Lipschitz, whereas we found two examples - travelling waves and self-similar solutions - where the pressure is Lipschitz, and has linear growth at the free boundary. The goal of the section is to prove that this fact is general.

Using Proposition 2.3, we may put our solution $u$ between two self-similar solutions - say, a $z$-translate of $\phi_{1-\varepsilon}$ and a $z$-translate of $\phi_{1+\varepsilon}$ - and infer that, for all $t \geqslant 0$, the function $z \mapsto u(t, z, 0)$ is compactly supported in $\mathbb{R}^{+}$. Therefore there is a function $\zeta(t)$ such that $u(t, z, x)>0$ for $z<\zeta(t)-$ we have $u(t, z, 0)>0$ for $z<\zeta(t)$, then this property extends to $x \neq 0$ by the strong maximum principle - and $u(t, z, x)=0$ for $z>\zeta(t)$.

The plan of the section is the following: first, we prove that the interface is Lipschitz, and that $p$ is globally Lipschitz. Then we will pause in order to prove a class of Harnack inequalities, and conclude the section by the nondegeneracy of $p$. This section roughly corresponds to Ref. [8] in the study of the porous medium equation, and the results are qualitatively the same.

\section{1. $\zeta$ and $p$ are Lipschitz}

Our first task is to prove that, for all $t_{0}>0, u$ is continuous at $\left(t_{0}, \zeta\left(t_{0}\right), 0\right)$. We state it under the

Proposition 4.1. - Choose any $t_{0}>0$. Then $u\left(t_{0}, \zeta\left(t_{0}\right)^{+}, 0\right)=u\left(t_{0}, \zeta\left(t_{0}\right)^{-}, 0\right)=0$.

Proof. - Assume this is not true and set $M:=u\left(t_{0}, \zeta\left(t_{0}\right)^{-}\right)>0$. Then, for all $t \geqslant t_{0}$ we have $u\left(t, \zeta\left(t_{0}\right), 0\right) \geqslant M$. From Proposition 2.4 we have

$$
u(t, z, x) \geqslant \phi_{M}\left(\frac{z-\zeta\left(t_{0}\right)}{\sqrt{t-t_{0}}}, \frac{x}{\sqrt{t-t_{0}}}\right) .
$$

Hence, for all $h>0$, we have 


$$
\zeta\left(t_{0}+h\right)-\zeta\left(t_{0}\right) \geqslant \zeta_{M}(t)=M z_{1} \sqrt{h}
$$

On the other hand, setting $x=0$ in the hyperbolic inequation (2.8) of Proposition 2.7, we have $u\left(t_{0}+h, z, 0\right) \leqslant u\left(t_{0}, z-C h, 0\right)$ which implies

$$
\zeta\left(t_{0}+h\right) \leqslant \zeta\left(t_{0}\right)+C h
$$

contradicting (4.1) for small $h>0$.

For all $\alpha \in\left[0,1\left[\right.\right.$, and for all $t \geqslant 0$, let $\zeta^{\alpha}(t)$ the minimal $z$ such that $u(t, z, 0)=\alpha$. Because $u$ is nonincreasing in $z$ and nondecreasing in $t$, we have $u(t, z, 0)>\alpha$ for all $z<\zeta^{\alpha}(t)$. Let us call the set $\left\{\left(t, \zeta^{\alpha}(t)\right\}\right.$ the $\alpha$-level line of $u(t, z, 0)$.

Proposition 4.2. - The function $\zeta$ is Lipschitz with constant $C$, as are all level lines of $u$.

Proof. - Same argument as above: for all $t_{0}>0$ and $h>0$ we have $u\left(t_{0}+h, z, 0\right) \leqslant$ $u\left(t_{0}, z-C h, 0\right)$; hence $\zeta^{\alpha}\left(t_{0}+h\right) \leqslant \zeta\left(t_{0}\right)+C h$. We conclude by noticing that $\zeta^{\alpha}$ is nondecreasing.

And finally we have the

PROPOSITION 4.3. - The function $p$ is Lipschitz in all its variables.

Proof. - Choose $\alpha \in\left[0, \frac{1}{2}\right]$ and let $\left(t_{0}>0, z_{0}\right)$ be such that $u\left(t_{0}, z_{0}, 0\right)=\alpha$. We wish to prove that $u_{z}\left(t_{0}, z_{0}, 0\right)$ is above a constant under control. For this, we first claim the existence of $r\left(t_{0}\right)>0$, controlled from below, such that the set

$$
Z\left(t_{0}\right):=\left\{z \in \mathbb{R}: \frac{1}{4} \leqslant u\left(t_{0}, z, 0\right) \leqslant \frac{1+\alpha}{2}\right\}
$$

is an interval of length at least $r\left(t_{0}\right)$. Let us assume this is not so, and let us consider any smooth $\varepsilon$-approximation of $u$, called $u^{\varepsilon}$. Then the set $Z^{\varepsilon}$ corresponding to $Z$ for $u^{\varepsilon}$ is an interval of length $r^{\varepsilon}$ going to 0 as $\varepsilon \rightarrow 0$. Then, still form Proposition 2.4, we have

$$
u^{\varepsilon}(t, z, 0) \geqslant \phi_{\frac{1+\alpha}{2}}\left(\frac{z-z_{0}}{\sqrt{t-t_{0}}}, 0\right) .
$$

The $\frac{1}{2}$ level line of $\phi_{\frac{1+\alpha}{2}}\left(\frac{z}{\sqrt{t}}, 0\right)$ is a graph $\left\{\left(t, \frac{1+\alpha}{2} z_{1 / 2} \sqrt{t}\right)\right\}$, with $z_{1 / 2}>0$. Now, we argue just as above: for small $h>0$ we have $u\left(t_{0}+h, z_{0} \frac{1+\alpha}{2} z_{1 / 2} \sqrt{h}+\mathrm{O}\left(r^{\varepsilon}\right), 0\right) \geqslant \frac{1}{2}$; on the other hand, due to Proposition 2.7, we have $u^{\varepsilon}\left(t_{0}+h, z_{0}+C h, 0\right) \leqslant \frac{1}{4}$. Sending $\varepsilon$ to 0 yields a contradiction.

From Proposition 2.7 once again, we infer the existence of $\tau_{0} \in\left[0, t_{0}\left[\right.\right.$, such that $t_{0}-\tau_{0}$ is controlled from below, and such that $u\left(\tau_{0}, z_{0}, 0\right) \geqslant \frac{1}{3}$. Arguing as above, we obtain that the set $Z\left(\tau_{0}\right)$ is also an interval of length at least $r\left(\tau_{0}\right)$, this last quantity being also under control. Therefore we may apply Proposition 2.8, and infer that $u$ is under control.

To prove our proposition, we only have to prove the same property for all values $\beta$ of $u$ between 0 and $\frac{1}{2}$. Let us choose such a $\beta$ and pick $h>0$ be such that $\alpha:=h \beta \in\left[0, \frac{1}{2}[\right.$. Then we remark that, due to the homogeneity considerations of Section 2.1, the function 
$p_{h}$ defined by

$$
p_{h}(\tau, \eta, \xi):=\frac{1}{h} p\left(t_{0}+h \tau, z_{0}+h \eta, \sqrt{h} \xi\right)
$$

is such that its square root satisfies the original problem (1.2). Moreover, its Lipschitz constant is the same as that of $p$. We now only have to repeat the above argument.

\subsection{Harnack inequalities for $-p_{z}$ and $p_{t}$}

In parabolic equations, Harnack inequalities are often established directly, for they are one possible step towards Hölder estimates. Another way to obtain them - which we choose in this paragraph - is to establish the Hölder estimates first, then a strong maximum principle.

The situation is the following. Let us pick $\left(t_{0}, z_{0}\right) \in \mathbb{R}_{+}^{*} \times \mathbb{R}_{+}^{*}$ and a small $h>0$. We consider the rescaled function $p$ :

$$
p(t, z, x):=\frac{1}{h} p\left(t_{0}+h t, z_{0}+h z, \sqrt{h} x\right) .
$$

We assume, for commodity, that $p(0,0,0)=1$. The above paragraph enables us to find $r>0$, controlled from below, such that

$$
p(t, z, 0) \geqslant \frac{1}{2} \text { for }-3 r \leqslant t, z \leqslant 3 r .
$$

Let us set $u:=\sqrt{p}$. We may arrange that (4.4) is also valid for any smooth $\varepsilon$-approximation of $u$, with $\varepsilon>0$ small enough. Let us finally set $v:=u_{t}$ or $u_{z}$. Then $v$ solves

$$
\left\{\begin{aligned}
v_{t}-v_{x x} & =0, & & (t, z, x) \in \mathbb{R}_{+} \times \mathbb{R}_{+}^{2}, \\
v_{x} & =(u w)_{z} & & (t>0, z \in \mathbb{R}, x=0) .
\end{aligned}\right.
$$

Proposition 4.4 (Compactness). - Choose $\alpha \in] 0, \frac{1}{2}[$. There exists $C>0$, independent of $h$, such that

$$
\left\|u_{z}\right\|_{C^{\alpha, \alpha / 2}\left([-r, r]^{2} \times \mathbb{R}_{+}\right)}+\left\|u_{t}\right\|_{C^{\alpha, \alpha / 2}\left([-r, r]^{2} \times \mathbb{R}_{+}\right)} \leqslant C .
$$

Eq. (4.6) is also valid for any smooth $\varepsilon$-approximation of $u$, with a constant $C$ independent of $h$ and $\varepsilon$.

Proof. - Let $\left(u^{\varepsilon}\right)_{\varepsilon>0}$ be a smooth $\varepsilon$-approximation of $u$; inequality (4.6) will be proved for $u^{\varepsilon}$. This will imply its validity for $u$.

We drop the superscript ${ }^{\varepsilon}$. Let us set

$$
\tilde{u}(t, z, x)=\int_{z}^{+\infty} u\left(t, z^{\prime}, x\right) \mathrm{d} z^{\prime} .
$$

Then $\tilde{u}$ satisfies the same problem as $u$ in $\mathbb{R}_{+}^{2}$, but the boundary condition at $x=0$ becomes

$$
\tilde{u}_{x}=-\frac{1}{2} u \tilde{u}_{z}
$$


We now notice that $\tilde{u}_{z}(0, .,$.$) belongs to C^{2, \alpha}\left(\mathbb{R}_{+}^{2}\right)$. Let $\gamma(t, z)$ be a cut-off function, equal to 1 in $[-r, r]^{2} \times \mathbb{R}_{+}$and to 0 outside $[-2 r, 2 r]^{2} \times \mathbb{R}_{+}$. Set $w=\gamma \tilde{u}$; we have

$$
\left\{\begin{aligned}
w_{t}-w_{x x} & =\gamma_{t} \tilde{u}, & & (t, z, x) \in \mathbb{R}_{+} \times \mathbb{R}_{+}^{2}, \\
w_{x} & =-\frac{1}{2} u\left(w_{z}-\gamma_{z} u\right) & & (t>0, z \in \mathbb{R}, x=0) .
\end{aligned}\right.
$$

Now, we may apply word by word the arguments of the proof of Proposition 2.9, because:

- Lemma 2.5 applies, due to the fact that the Lipschitz constant of $u$ is controlled on $[-3 r, 3 r]^{2} \times \mathbb{R}_{+}$,

- the term that may cause a bootstrap failure due to the possible lack of smoothness of $u_{0}$, see Remark 2.1, namely:

$$
\int_{0}^{t} e^{(t-s) \partial_{x x}^{2}} \gamma_{t} \tilde{u} \mathrm{~d} s
$$

is now controlled by $\tilde{u}(t, z, 0)$ and $e^{t \partial_{x x}^{2}} \tilde{u}_{0}$, which has now $C^{2+\alpha, 1+\alpha / 2}$ estimates in $t$ and $z$.

The bound for $u$ now directly results from Proposition 2.9.

PROPOSITION 4.5 (Strong maximum principle for $v$ ). - Assume that $v$ is not identically 0 in the box $B_{r}(0,0) \times \mathbb{R}_{+}$. Then $v>0$ in $B_{r}(0,0) \times \mathbb{R}_{+}$.

Proof. - The boundary condition for $v$ reads

$$
v_{x} \leqslant u v_{z} .
$$

We will prove that the set $\{v>0\}$ is closed in $B_{r}(0,0) \times \mathbb{R}_{+}$. From the usual strong maximum principle for the heat equation, it is enough to prove that the subset $\{(t, z) \in$ $\left.B_{r}(0,0): v(t, z, 0)>0\right\}$ is closed in $B_{r}(0,0)$. Let $\left(t_{n}, z_{n}\right)_{n} \geqslant 2$ be a sequence converging to $\left(t_{0}, z_{0}\right)$ such that $v\left(t_{n}, z_{n}, 0\right)>0$.

Case 1. We have $t_{n}>t_{0}$ and there is $\left.\rho \in\right] 0, r-\left|z_{0}\right|\left[\right.$ and $\left.t_{1} \in\right] t_{0}-r, t_{0}[$ such that, for all $z \in\left[z_{0}-\rho, z_{0}+\rho\right]$ and $\left.\tau \in\right] t_{1}, t_{0}[$, we have: $v(t, z, 0)=0$. Then we have, from the strong maximum principle for the heat equation:

$$
\forall(z, x) \in\left[z_{0}-\rho, z_{0}+\rho\right] \times \mathbb{R}_{+}: v\left(t_{1}, z, 0\right)=0 .
$$

For $n$ large enough, we have $\left.z_{n} \in\right] z_{0}-\rho, z_{0}+\rho[$. We may assume, without loss of generality, that $z_{n}<z_{0}$. Let us choose such a large $n$; then $v(t, z, x) \geqslant w(t, z, x)$, where

$$
\begin{aligned}
w_{t}-w_{x x} & =0, & & (t, z, x) \in] t_{1}, t_{n}[\times] z_{n}, z_{0}+r\left[\times \mathbb{R}_{+},\right. \\
w_{x} & =-\frac{1}{2} w_{z} & & \left(t_{1} \leqslant t \leqslant t_{n}, z_{n} \leqslant z \leqslant z_{0}+r, x=0\right), \\
w\left(t, z_{n}, 0\right) & =\varphi_{n}(t), & & w\left(t, z_{0}+\rho, 0\right)=0, \\
w\left(t_{1}, z, x\right) & =0, & &
\end{aligned}
$$


where $\varphi_{n}(t)$ is any smooth nondecreasing function on $\left[t_{1}, t_{n}\right]$ such that $\varphi_{n}\left(t_{n}\right)=$ $v\left(t_{n}, z_{n}, 0\right)$. To see that $w$ and $v$ indeed compare, we only have to compare $w$ with the derivative of a smooth $\varepsilon$-approximation of the original function $u$; then we notice that we have $w_{z} \leqslant 0$ - if we had assumed $z_{n}>z_{0}$, we would have had to choose the boundary condition $w_{x}=w_{z}$ and $w_{z}$ would have been nonnegative. Eq. (4.11) implies, among other things, that we have $w=I_{1 / 2} w_{z}$, for all $t \in\left[t_{1}, t_{n}\right]$ and $\left.z \in\right] z_{n}, z_{0}+\rho[$. Hence we have

$$
w_{t}-w_{z z}=0 \quad \text { on }\left\{t \in\left[t_{1}, t_{n}\right], z \in\right] z_{n}, z_{0}+\rho[\}
$$

with the above-mentioned boundary conditions. The strong maximum principle for the heat equation implies $w>0$ on $] t_{1}, t_{n}\left[\right.$; hence $v\left(t_{0}, z_{0}, 0\right)>0$.

Case 2. The sequence $\left(t_{n}, z_{n}\right)$ may be assumed to satisfy $t_{n}<t_{0}$. Then, for large enough $n \geqslant 2$ there is $\delta_{n}>0$ and $\rho_{n}>0$ such that $v\left(t_{n}, z, 0\right)>\delta_{n}$ for $z_{n}-2 \rho_{n} \leqslant z \leqslant$ $z_{n}+2 \rho_{n}$. Let us consider the function $w_{n}$, satisfying

$$
\begin{aligned}
\left(\partial_{t}-\partial_{x x}\right) w_{n} & =0 & & (t, z, x) \in] t_{n},+\infty[\times] z_{n}-\rho_{n}, z_{n}+\rho_{n}\left[\times \mathbb{R}_{+},\right. \\
\partial_{x} w_{n} & =\partial_{z} w_{n} & & \left(t \geqslant t_{n}, z_{n}-\rho_{n} \leqslant z \leqslant z_{n}+\rho_{n}, x=0\right), \\
w_{n}\left(t, z_{n}-\rho_{n}, 0\right) & =0 & & \left(t>t_{n}\right), \\
w_{n}\left(t, z_{0}+\rho_{n}, 0\right) & =0 & & \left(t>t_{n}\right), \\
w_{n}\left(t_{n}, z, 0\right) & =\delta_{n} & & \left(z_{n}-\rho_{n}, z<z+\rho_{n}\right) .
\end{aligned}
$$

Set $\tilde{w}_{n}(t, z):=w_{n}(t, z, 0)$. Then $w_{n}$ is symmetric about $z_{n}$ and we have - examine the function $w-\delta_{n}$ :

$$
\begin{aligned}
\left(\partial_{t}-\partial_{z z}\right) \tilde{w}_{n} & =0 \quad(t, z, x) \in] t_{n},+\infty[\times] z_{n}-\rho_{n}, z_{n}+\rho_{n}[ \\
\tilde{w}_{n}\left(t, z_{n}-\rho_{n}, 0\right) & =0 \\
\tilde{w}_{n}\left(t, z_{0}+\rho_{n}, 0\right) & =0 \\
\tilde{w}_{n}\left(t_{1}, z, 0\right) & =\delta_{n} .
\end{aligned}
$$

We define a subsolution $w(t, z, x)$ to Eqs. (4.5), (4.10) by setting

$$
w(t, z, x)=\left\{\begin{array}{cc}
\tilde{w}_{n}\left(t, \frac{z+z_{n}}{2}, x\right) & \text { for } z \leqslant z_{n}, \\
\tilde{w}_{n}(t, z, x) & \text { for } z \geqslant z_{n} .
\end{array}\right.
$$

From the definition of $\tilde{w}$ we have $w(t, z, x)>0$ for $t \geqslant t_{n}, z_{n}-2 \rho_{n} \leqslant z \leqslant z_{n}+\rho_{n}$. If $\left.z_{0} \in\right] z_{n}-2 \rho_{n}, z_{n}+\rho_{n}$ [, we are done. If not, we have proved the existence of $\varepsilon_{n} \leqslant \delta_{n}$ such that

$$
\forall t \in\left[t_{n}, t_{0}+r\right], \quad v\left(t, z_{n}, 0\right) \geqslant \varepsilon_{n} .
$$


Without loss of generality, we may once again assume $z_{n}<z_{0}$. Let $\psi_{1 / 2}(z, x)$ be the linear self-similar solution of Section 2.3 with $b=\frac{1}{2}$. From Proposition 3.2 we may find $\tau_{n}$ and $\lambda_{n}>0$, both going to 0 as $n \rightarrow+\infty$, such that

- For all $t \in\left[t_{n}, t_{0}+\tau_{n}\right]$, we have

$$
\psi_{1 / 2}\left(\frac{r-z_{n}}{\sqrt{t_{0}+\tau_{n}-t_{n}}}, 0\right) \leqslant \lambda_{n}
$$

- we have

$$
\psi_{1 / 2}\left(\frac{z_{0}-z_{n}}{\sqrt{t_{0}-t_{n}}}, 0\right)>\lambda_{n}
$$

This implies

$$
v(t, z, x) \geqslant \varepsilon_{n}\left(\psi_{1 / 2}\left(\frac{z-z_{n}}{\sqrt{t-t_{n}}}, \frac{x}{\sqrt{t-t_{n}}}\right)-\lambda_{n}\right)
$$

as a consequence we have $v\left(t_{0}, z_{0}, 0\right)>0$.

Combining the two propositions leads us to the main result of this paragraph.

THEOREM 4.1 (Harnack inequalities). - Let $0<r<1$ and a small $\varepsilon>0$ be given. There exists $C_{\varepsilon}>0$, independent of $h$, see the definition of $v$ from $E q$. (4.3) above, such that

$$
\sup _{(t, z) \in B_{r}^{+}(-\varepsilon, 0)} v(t, z, 0) \leqslant C_{\varepsilon} \inf _{(t, z) \in B_{r}^{+}(\varepsilon, 0)} v(t, z, 0) .
$$

Proof. - Assume the existence of a sequence of initial data $\left(u_{0}^{n}\right)_{n}$ for the Cauchy problem (1.2), such that the corresponding function $v_{n}$ satisfies

$$
\sup _{(t, z) \in B_{r}^{+}(-\varepsilon, 0)} v_{n}(t, z, 0)=1, \quad \lim _{n \rightarrow+\infty} \inf _{(t, z) \in B_{r}^{+}(\varepsilon, 0)} v_{n}(t, z, 0)=0 .
$$

By compactness, Proposition 4.4, the sequence $\left(v_{n}\right)_{n}$ converges in $C^{\alpha, \alpha / 2}\left(B_{r+\varepsilon}^{+}(0,0)\right)$ to a function $v$, whose supremum in $B_{r}^{+}(-\varepsilon, 0)$ is 1 , and whose infimum is 0 . This contradicts the strong maximum principle.

\subsection{Nondegeneracy}

The statement is

THEOREM 4.2. - Let $0<\delta<T$ be chosen. There exists $\gamma(\delta)>0$ such that, for all $t \in[0, T]$ and $z \in[0, \zeta(t)[$, we have

$$
\begin{aligned}
& -p_{z} \geqslant \gamma(\delta), \quad p_{t} \geqslant \gamma(\delta), \\
& \dot{\zeta}(t) \geqslant \gamma(\delta) .
\end{aligned}
$$

To prove it, we first show, as in [8], that the speed of the interface is controlled from below; then we conclude by comparison with a self-similar solution. 
Lemma 4.1. - Choose $T>0$. There exist a positive constant $B$, depending on $T$, such that

$$
(2 t+B) u_{t}+\left(z-\zeta_{0}\right) u_{z}+u \geqslant 0
$$

on $[0, T] \times \Gamma$, in the distributional sense.

Proof. - In Proposition 2.1 we take $c=\frac{1}{1+\varepsilon}$ and $b=(1+\varepsilon)^{2}$. Let us consider a large $B>0$, to be chosen later. The function

$$
u_{B}(\varepsilon, t, z, x):=(1+\varepsilon) u\left((1+\varepsilon)^{2} t+B \varepsilon,(1+\varepsilon)\left(z-\zeta_{0}\right),(1+\varepsilon) x\right)
$$

is a solution of (1.2), and the function

$$
\begin{aligned}
v(t, z, x) & :=\left.\frac{\partial}{\partial \varepsilon}\left(u_{B}(\varepsilon, .)-u\right)\right|_{\varepsilon=0}(t, z, x) \\
& =(2 t+B) u_{t}+x u_{x}+\left(z-\zeta_{0}\right) u_{z}+u
\end{aligned}
$$

satisfies the $(t, x)$ heat equation inside the domain, with the linearized boundary condition

$$
v_{x}=\frac{1}{2} \partial_{z}(u v) .
$$

We are going to prove that, if $B$ is large enough, then $v(0,) \geqslant$.0 on the domain $D=\left\{z \leqslant \zeta_{0}, 0 \leqslant x \leqslant 2 \sqrt{2 T}\right\}$ and on the strip $S=\left\{t>0, z \leqslant \zeta_{0}, x=2 \sqrt{2 T}\right\}$. This will imply $v(t, z, 0) \geqslant 0$ - same argument as in Section 2.1. The assumptions on $u_{0}$ gaurantee the existence of $M(T)$ such that

$$
-x \partial_{x} u_{0} \leqslant \frac{u_{0}}{2}+M \partial_{x x} u_{0} .
$$

Hence the nontrivial task is to prove that $v \geqslant 0$ on $S$. Since we have $\left(z-\zeta_{0}\right) u_{z} \geqslant 0$ for $z \leqslant \zeta_{0}$, we only have to care about controlling $x u_{x}$ by a portion of $u$ and a large multiple of $u_{x x}$ at $x=1$, for all $t \leqslant T$ and $z \leqslant \zeta_{0}$. Let us write

$$
u=e^{t \partial_{x x}} u_{0}-\int_{0}^{t} e^{(t-s) \partial_{x x}} p_{z} \delta_{x=0} \mathrm{~d} s:=u_{1}+u_{2} .
$$

1. Let us first examine $u_{1}$. If $z \leqslant 0$, there is nothing to prove. If $0 \leqslant z<\zeta_{0}$, then we write

$$
u_{1}(t, z, 2 \sqrt{2 T})=\frac{\sqrt{\zeta_{0}-z}}{\sqrt{4 \pi t}} \int_{-\infty}^{+\infty} e^{-(2 \sqrt{2 T}-y)^{2} / 4 t} f(z, y) \mathrm{d} y .
$$

This region is cut into two pieces: first, there exists $\delta(T)>0$ such that, for all $z \in[0, \delta]$ and all $x \in[0,2 \sqrt{2 T}]$ we have:

$$
-x \partial_{x} u_{0}(z, x) \leqslant \frac{1}{4} u_{0}(z, x),
$$


simply because $u_{0}$ is close to 1 and $\partial_{x} u_{0}$ close to 0 . On $\left[\delta, \zeta_{0}\right] \times[0,2 \sqrt{2 T}]$, because $f_{x x}$ is nonnegative and nonzero, there exist $\mu_{1}(T)>0, \mu_{2}(T)>0$ such that, for all $t \leqslant T$ :

$$
\int_{-\infty}^{+\infty} e^{-(2 \sqrt{2 T}-y)^{2} / 4 t} f_{x x}(z, y) \mathrm{d} y \geqslant \mu_{1}(T), \quad \int_{-\infty}^{+\infty} e^{-(2 \sqrt{2 T}-y)^{2} / 4 t} f_{x}(z, y) \mathrm{d} y \geqslant \mu_{2}(T) .
$$

Hence we have, for all $(t, z) \in[0, T] \times\left[0, \zeta_{0}\right]$ :

$$
-x \partial_{x} u_{1}(t, z, 1) \leqslant \frac{\mu_{2}(T)}{\mu_{1}(T)} \partial_{x x} u_{1}(t, z, 1) .
$$

2. We have, because $p_{z} \leqslant 0$ :

$$
\begin{aligned}
\partial_{x x} u_{2}(t, z, 2 \sqrt{2 T}) & =-\int_{0}^{t} \frac{e^{-2 T /(t-s)}}{4 \sqrt{\pi}(t-s)^{3 / 2}}\left(\frac{4 T}{(t-s)}-1\right) p_{z}(s, z, 2 \sqrt{2 T}) \mathrm{d} s \\
& \geqslant-\frac{3}{4 \sqrt{\pi}} \int_{0}^{t} \frac{e^{-2 T /(t-s)}}{(t-s)^{3 / 2}} p_{z}(s, z, 2 \sqrt{2 T}) \mathrm{d} s .
\end{aligned}
$$

On the other hand, we have

$$
\begin{aligned}
-2 \sqrt{2 T} \partial_{x} u_{2}(t, z, 2 \sqrt{2 T}) & =-8 T \int_{0}^{t} \frac{e^{-2 T /(t-s)}}{2 \sqrt{\pi}(t-s)^{3 / 2}} p_{z}(s, z, 2 \sqrt{2 T}) \mathrm{d} s \\
& \leqslant \frac{16 T}{3} \partial_{x x} u_{2}(t, z, 2 \sqrt{2 T}) .
\end{aligned}
$$

Gathering (4.18), (4.20) and (4.21), we take

$$
B \geqslant \max \left(\frac{8 T}{3}, \frac{\mu_{2}(T)}{\mu_{1}(T)}, M\right)
$$

We obtain the desired result.

Lemma 4.1 has an immediate corollary, just as in the porous medium equation: as soon as the free boundary starts moving, it will keep moving for all later time. Here is the precise statement.

COROLlaRY 4.1 ([8], Corollary 3.2). - Choose $\delta>0$ and assume the existence of $t_{\delta}>0$ for which $\zeta\left(t_{\delta}\right)=\zeta_{0}+\delta$. There exists $\mu_{\delta}>0$ such that, for all $t \geqslant t_{\delta}$, we have $\dot{\zeta}(t) \geqslant \mu_{\delta}$.

Proof. - Choose $t_{0} \geqslant \delta$; we have $\zeta\left(t_{0}\right) \geqslant \delta$. From Lemma 4.1 we have, for all $t \in$ $\left[\delta, t_{0}+1\right]$ :

$$
u_{t}+A\left(\left(z-\zeta_{0}\right) u_{z}+u\right) \geqslant 0
$$

with $A=1 /\left(2 t_{0}+B+2\right)$. Therefore we have, for all $(t, z) \in\left[t_{0}, t_{0}+1\right]$ :

$$
u(t, z, 0) \geqslant e^{-A\left(t-t_{0}\right)} u\left(t_{0}, Z(t, z), 0\right),
$$


where

$$
Z(t, z)=\zeta_{0}+\left(z-\zeta_{0}\right) e^{-A\left(t-t_{0}\right)} .
$$

For $z=\zeta\left(t_{0}\right)$, the first instant $t_{h}$ such that we have $Z(t, z)=\zeta\left(t_{0}\right)-A \delta h$ is given by

$$
t_{h}-t_{0}=-\frac{1}{A} \log \left(1-\frac{\delta h}{\zeta\left(t_{0}\right)-\zeta_{0}}\right) \leqslant-\frac{1}{A} \log (1-A h) \leqslant h .
$$

As a consequence we have $\zeta\left(t_{0}+h\right)-\zeta\left(t_{0}\right) \geqslant A \delta h$, hence $\dot{\zeta}\left(t_{0}\right) \geqslant A \delta$.

Proof of Theorem 4.2. - In order to prove that the speed of the interface is controlled from below, we have to show that the interface starts moving immediately. Once this is done, the nondegeneracy result will follow from comparison with low speed self-similar solutions.

To prove that the free boundary starts moving at once, let us pick any small $\delta>0$; because $p_{0}$ decays linearly in a vicinity of $\zeta_{0}$ there exists $q>0$ such that $p\left(0, \zeta_{0}-\delta, 0\right) \geqslant$ $q \delta$. Hence we have

$$
p(t, z, x) \geqslant \phi_{\sqrt{q \delta}}^{2}\left(\frac{z-\zeta_{0}-\delta}{\sqrt{t}}, \frac{x}{\sqrt{t}}\right)
$$

this implies

$$
\zeta(t) \geqslant \zeta_{0}-\delta+\sqrt{q \delta} z_{1} \sqrt{t}
$$

hence we have, from (3.8): $\zeta(t)>\zeta_{0}$ as soon as $t \geqslant \sqrt{\delta} / \sqrt{q} z_{1}$, and we conclude with the arbitrariness of $\delta$.

Let us now prove that, for all $\delta>0$ we have

$$
\inf _{t \in[\delta, T]} \liminf _{h \rightarrow 0^{+}}\left(\frac{1}{h} p(t, \zeta(t)-h, 0)\right)>0 .
$$

If this were not true, there would exist a sequence $\left(t_{n}\right)_{n}$, going to some $t_{0}>0$, a sequence $\left(h_{n}\right)_{n}$ going to 0 , such that

$$
\lim _{n \rightarrow+\infty} \frac{1}{h_{n}} p\left(t_{n}, \zeta\left(t_{n}\right)-h_{n}, 0\right)=0 .
$$

Set

$$
p_{n}(\tau, \eta, \xi)=\frac{1}{h_{n}} p\left(t_{n}+h \tau, \zeta\left(t_{n}\right)+h_{n} \eta, \sqrt{h_{n}} \xi\right) ;
$$

$p_{n}$ satisfies the same problem as $p$. Set $\varepsilon_{n}=p_{n}(0,-1,0)$; because of Proposition 2.7 there exists $\tau_{n}>0$, bounded away from 0 with respect to $n$, such that

$$
p_{n}\left(\tau_{n},-1,0\right)=2 \varepsilon_{n} .
$$

Hence we have, for all $\tau \leqslant \tau_{n}$ :

$$
p_{n}(\tau, \eta, 0) \leqslant \phi_{\sqrt{2 \varepsilon_{n}}}\left(\frac{\eta-\zeta\left(t_{n}\right)}{\sqrt{\tau}}, \frac{\sqrt{h}_{n} \xi}{\sqrt{\tau}}\right) .
$$


Inequations (4.27) and (3.8) imply $\zeta\left(\tau_{n}\right) \leqslant \sqrt{2 \varepsilon_{n} \tau_{n}} z_{1}$; on the other hand, because $\dot{\zeta}$ is bounded away from 0 we have the existence of $\mu>0$ such that $\dot{\zeta} \geqslant \mu$. Hence $\zeta\left(\tau_{n}\right) \geqslant \mu \tau_{n} ;$ a contradiction.

The nondegeneracy of $p$ follows from (4.25). We first note that, for every $t_{0}>0$ and $h>0$, there exists $q>0$, bounded away from 0 independently of $h$ and $t_{0}$, such that, if we have

$$
p\left(t_{0}, \zeta_{h}^{1}\left(t_{0}\right), 0\right)=h, \quad p\left(t_{0}, \zeta_{h}^{2}\left(t_{0}\right), 0\right)=\frac{h}{2}
$$

then $\zeta_{h}^{2}\left(t_{0}\right)-\zeta_{h}^{1}\left(t_{0}\right)=q h$. If this were not so, we would contradict, after setting the $z$ origin at $\zeta\left(t_{0}\right)$ and rescaling, the fact that $p$ is Lipschitz. Hence there is a point in the segment $\left[\zeta_{h}^{1}\left(t_{0}\right), \zeta_{h}^{2}\left(t_{0}\right)\right]$ such that $p_{z} \geqslant q / 2$ at that point. Rescaling and applying the Harnack inequalities, we obtain a control from below for $-p_{z}$ in the segment. Because of the arbitrariness of $h$, this control is valid everywhere.

Finally, a lower bound on $p_{t}$ in the vicinity of the free boundary is obtained from inequality (4.16). Away from the free boundary, this is once again due to the Harnack inequalities.

\section{The free boundary is $C^{1, \alpha}$}

This section is devoted to the application of the iterative technique of [9] in the context of Problem (1.2), whose idea is briefly recalled now. Because $p_{t}$ and $-p_{z}$ are controlled from below in a vicinity of the free boundary, there is a common cone of directions of the $(t, z, x)$-space along which the function $p$ is increasing - in the same neighbourhood of the free boundary. The basic iteration consists in enlarging this cone by proving that, if $p$ is nondecreasing along a direction $v$, then $p_{v}$ is in fact controlled from below. This relies on the Harnack inequalities of Section 4, as well as on a separation argument of the free boundaries of two solutions of (1.2).

Then we rescale and iterate the argument; as we move closer to the free boundary the common cone of monotonicity tends to be a whole half-space: this implies the differentiablity of the free boundary. Further, the cone enlargement being controlled at each step, the argument yields in fact the Hölder continuity of $\dot{\zeta}$ as well as $p_{t}$ and $p_{z}$.

What will be explained in this section is the construction of sub-solutions - that slightly differ from [9] - then the basic iteration. The rest of the argument is exactly as in [9], Section 4.

In the whole section, the problem will be examined on a time interval $\left[t_{\min }, T\right]$ where $t_{\min }>0$ is a little larger than the minimal time required by the free boundary to start moving. Hence all the constants in the next section will depend on $t_{\min }$, but we will not mention this dependence.

\subsection{Sub-solutions}

Assume the free boundary of $u$ in the $(t, z)$-plane to be located at the point $(t=0, z=$ $0)$. Let us denote by $l(t, z, x$,$) a travelling wave with speed V_{0}$.

LEMMA 5.1. - Assume that the interfaces of $l$ and $u$ coincide at time $t=0$ and $V_{0}<\dot{\zeta}(t)$ for $-1 \leqslant t \leqslant 1$. 
Pick two positive numbers $\delta$ and $\varepsilon$. There exist $\delta_{0}>0, \varepsilon_{0}>0$ such that, if $\delta \leqslant \delta_{0}$ and $\varepsilon \leqslant \varepsilon_{0}$, then the function

$$
\underline{u}_{1}^{\varepsilon}=u+\varepsilon(u-\delta l)^{+}
$$

is a sub-solution to (1.2) for $-1 \leqslant t \leqslant 0$.

Proof. - From the convexity of $X \mapsto X^{+}$, we have $\left(\partial_{t}-\partial_{x x}\right) \underline{u}_{1}^{\varepsilon} \leqslant 0$. As for the boundary condition, there is nothing to prove if $u \leqslant \delta l$. On the other hand, if $u \geqslant \delta l$, we have

$$
\begin{aligned}
\partial_{x} \underline{u}_{1}^{\varepsilon}-\underline{u}_{1}^{\varepsilon} \partial_{z} \underline{u}_{1}^{\varepsilon}= & -\varepsilon(1+\varepsilon) u u_{z}-\varepsilon \delta(1+\varepsilon \delta) l l_{z} \\
& +\varepsilon \delta(1+\varepsilon)\left(u l_{z}+l u_{z}\right) .
\end{aligned}
$$

We distinguish two cases.

Case $1 .(1+\varepsilon) u \geqslant l$.

Then we use $u\left|u_{z}\right| \geqslant \delta l\left|u_{z}\right|$ and $l l_{z} \geqslant(1+\varepsilon) u\left|l_{z}\right|$. This implies

$$
\partial_{x} \underline{u}_{1}^{\varepsilon}-\underline{u}_{1}^{\varepsilon} \partial_{z} \underline{u}_{1}^{\varepsilon} \geqslant-\varepsilon^{2} \delta^{2} l l_{z} \geqslant 0 .
$$

Case 2. $(1+\varepsilon) u \leqslant l$.

First, we use $\delta l\left|u_{z}\right| \leqslant \delta(1+\varepsilon) u\left|u_{z}\right|$; second, we use Lemma 4.1 to infer the existence of $C>0$ such that $C\left|u_{z}\right| \geqslant\left|l_{z}\right|$. Hence we have $u\left|l_{z}\right| \leqslant C u\left|u_{z}\right|$, and we end up with

$$
\partial_{x} \underline{u}_{1}^{\varepsilon}-\underline{u}_{1}^{\varepsilon} \partial_{z} \underline{u}_{1}^{\varepsilon} \geqslant-\varepsilon^{2} \delta^{2} l l_{z}-\varepsilon(1-\delta(1+C))(1+\varepsilon) u u_{z},
$$

which implies the lemma as soon as we choose $\delta \leqslant \delta_{0}:=\frac{1}{1+C}$.

LEMmA 5.2. - There exists $\lambda>0$ such that, for all $\varepsilon \in] 0,1)$, the function $\underline{u}_{2}^{\varepsilon}$ given by

$$
\underline{u}_{2}^{\varepsilon}(t, z, x)=(1+\varepsilon) u(t+\varepsilon \lambda z, z, x)
$$

is a sub-solution to (1.2).

Proof. - Obviously, the heat equation inside $\mathbb{R}_{+}^{2}$ is satisfied. As for the boundary condition we have

$$
\begin{aligned}
\partial_{x} \underline{u}_{2}^{\varepsilon}-\underline{u}_{2}^{\varepsilon} \partial_{z} \underline{u}_{2}^{\varepsilon} & =(1+\varepsilon) u_{x}-(1+\varepsilon)^{2}\left(u u_{z}-\varepsilon \lambda u u_{t}\right) \\
& =-\varepsilon(1+\varepsilon) u u_{z}-\varepsilon \lambda(1+\varepsilon)^{2} u u_{t} .
\end{aligned}
$$

But we know that $-u_{t} \geqslant C u_{z}$; hence $\partial_{x} \underline{u}_{2}^{\varepsilon}-\underline{u}_{2}^{\varepsilon} \partial_{z} \underline{u}_{2}^{\varepsilon} \geqslant 0$ for $\lambda \leqslant \frac{1}{2 C}$.

\subsection{The iteration}

Let $\left(t_{0}, z_{0}\right)=\left(\zeta^{-1}\left(z_{0}\right), z_{0}\right)$ be a free boundary point. In order to initiate the sequence of iterations leading to the $C^{1, \alpha}$ estimate for $\zeta$ at $\left(t_{0}, z_{0}\right)$, we first notice that Theorem 4.2 has led us to the following situation. By translating and rescaling we may assume $\left(t_{0}, z_{0}\right)=(0,0)$. For all $\rho \in[0,1]$ let us consider the rectangle

$$
R_{0}(\rho)=\left\{\zeta^{-1}(-1) \leqslant t \leqslant \zeta^{-1}(1),-1-\rho \leqslant z \leqslant 1\right\} .
$$


We denote

- by $L$ a common upper bound for $p_{t}$ and $-p_{z}$ in $R_{0}$,

- and by $\gamma$ a common lower bound for $p_{t}$ and $-p_{z}$ in $R_{0}$.

Hence we have

$$
\forall t \in\left[\zeta^{-1}(-1), \zeta^{-1}(1)\right], \quad \frac{\gamma}{L} \leqslant \dot{\zeta}(t) \leqslant \frac{L}{\gamma} .
$$

Estimates (4.15) hold, in the box $B_{2 L / \gamma}(0,0)$ and, in particular, in $R_{0}(1)$. Let us examine the consequences of Lemmas 5.1 and 5.2.

PROPOSITION 5.1. - Let $\tilde{u}$ be a solution of (1.2) in $R_{0}(1) \times \mathbb{R}_{+}$such that, for some $\rho \in] 0,1[$ :

$$
\tilde{u} \geqslant u \quad \text { in } R_{0}(\rho) \times \mathbb{R}_{+}, \quad \tilde{u}(t,-1-\rho, 0) \geqslant(1+2 \varepsilon) u(t,-1-\rho, 0) .
$$

(i) There exists $\rho_{0}>0$ such that: if (5.7) holds for a given $\left.\left.\rho \in\right] 0, \rho_{0}\right]$, then we have

$$
\forall(t, z) \in\left[\zeta^{-1}\left(-\frac{1}{2}\right), \zeta^{-1}\left(\frac{1}{2}\right)\right] \times\left[-\frac{1}{2}, \frac{1}{2}\right], \quad \tilde{u}(t, z, 0) \geqslant(1+\varepsilon) u(t, z, 0) .
$$

(ii) Let $\rho>0$ be chosen as above. There holds

$$
\begin{aligned}
& \forall(t, z) \in\left[\zeta^{-1}\left(-\frac{1}{2}\right), \zeta^{-1}(1)+\zeta^{-1}\left(-\frac{1}{2}\right)\right] \times\left[-\frac{1}{2}, 1\right] \\
& \tilde{u}\left(t-\zeta^{-1}\left(-\frac{1}{2}\right), z+\frac{1}{2}, \alpha\right) \geqslant(1+\varepsilon) u\left(t-\zeta^{-1}\left(-\frac{1}{2}\right)+\varepsilon \lambda\left(z+\frac{1}{2}\right), z+\frac{1}{2}, x\right) .
\end{aligned}
$$

Proof. - 1. Choose $z_{0} \in\left[-\frac{1}{2}, \frac{1}{2}\right]$ and $t_{0}=\zeta^{-1}\left(z_{0}\right)$. Set

$$
V_{0}=\frac{1}{3\left(t_{0}-\zeta^{-1}(-1)\right)}
$$

it is controlled from above and below. Let us consider the travelling wave $l_{V_{0}}$ translated in such a way that its interface sits exactly at $z=-\frac{1}{3}$ at time $t=-1$; then, at $t=t_{0}$, its interface is located at $z=z_{0}$. From Lemma 5.1 the function $\underline{u}_{1}^{\varepsilon}=u+\varepsilon\left(u-\delta l_{V_{0}}\right)^{+}$is a subsolution to (1.2) as soon as $\delta>0$ is small enough. Let us consider such a $\delta$.

2. Let us prove the existence of $\rho_{\delta}>0$ such that: for all $\rho \leqslant \rho_{\delta}, \delta l_{V_{0}} \geqslant u$ on $\left\{t=\zeta^{-1}(-1),-1-\rho \leqslant z, x \geqslant 0\right\}$. We have:

- $0=u\left(\zeta^{-1}(-1), z, x\right) \leqslant \delta l_{V_{0}}$ on $[-1-\rho,+\infty] \times \mathbb{R}_{+}$,

- for $z \in[-1-\rho,-1]$ we have, from the Lipschitz property for $p$ :

$$
p\left(\zeta^{-1}(-1), z, 0\right) \leqslant L(1-z) \leqslant L \rho .
$$

Hence, for all $z \in[-1-\rho,-1]$ we have

$$
\begin{aligned}
\delta l_{V_{0}}\left(\zeta^{-1}(-1),-1-\rho, 0\right) & =\delta \sqrt{4 \pi V_{0}\left(1+\rho+V_{0} \zeta^{-1}(-1)\right)} \\
& \geqslant \delta \sqrt{\frac{8 \pi V_{0}}{3}} \quad \text { from }(5.10) .
\end{aligned}
$$

Hence the result as soon as $\rho \leqslant 8 \pi V_{0} /\left(3 L^{2}\right)$.

Therefore $\delta l_{V_{0}} \leqslant u$ on $\left[-1-\rho,+\infty\left[\times \mathbb{R}_{+}\right.\right.$at $t=-1$ as soon as $\rho>0$ is less than some $\rho_{\delta}>0$ whose value is given above. Let such a $\rho$ be chosen. 
3. Let us prove (i). Let us first notice that we have obviously:

$$
\underline{u}_{1}^{\varepsilon}(t,-1-\rho, 0) \leqslant(1+2 \varepsilon) u(t,-1-\rho, 0) \leqslant \tilde{u}(t,-1-\rho, 0)
$$

for $t \geqslant \zeta^{-1}(-1)$. Therefore from 1 and 2 we have $\underline{u}_{1}^{\varepsilon}(t, z, x) \leqslant \tilde{u}(t, z, x)$ for all $(t, z, x) \in$ $R_{0} \times \mathbb{R}_{+}$; hence at $t=t_{0}$ we have

$$
\forall z \leqslant z_{0}, \quad \tilde{u}\left(t_{0}, z, 0\right) \geqslant u\left(t_{0}, z, 0\right)+\varepsilon\left(u\left(t_{0}, z, 0\right)-l_{V_{0}}\left(t_{0}, z, 0\right)\right)^{+} .
$$

Moreover at $t=t_{0}$ the interfaces of $u$ and $l_{V_{0}}$ coincide. By nondegeneracy, we have $2 \delta l_{V_{0}}\left(t_{0}, z, 0\right) \leqslant u\left(t_{0}, z, 0\right)$ as soon as $\delta$ is small enough, and this fixes $\rho$ once and for all. We have therefore

$$
\left(u\left(t_{0}, z, 0\right)-\delta l_{V_{0}}\left(t_{0}, z, 0\right)\right)^{+}=u\left(t_{0}, z, 0\right)-\delta l_{V_{0}}\left(t_{0}, z, 0\right)^{+},
$$

and Property (i) holds.

4. Property (ii) is now almost immediate. Indeed, Lemma 5.2 asserts that

$$
\tilde{u}_{2}^{\varepsilon}(t, z, x):=(1+\varepsilon) u\left(t-\zeta^{-1}\left(-\frac{1}{2}\right), z+\frac{1}{2}, x\right)
$$

is a subsolution to (1.2). Moreover

- because $u_{t} \geqslant 0$, we have

$$
\tilde{u}_{2}^{\varepsilon}\left(\zeta^{-1}\left(\frac{1}{2}\right), z, x\right) \geqslant(1+\varepsilon) u\left(0, z+\frac{1}{2}, x\right)=0
$$

for all $(z, x) \in\left[-\frac{1}{2}, 1\right]$

- by assumption, we have, at $z=-\frac{1}{2}$ :

$$
\tilde{u}_{2}^{\varepsilon}(t, z, 0) \geqslant(1+\varepsilon) u\left(t-\zeta^{-1}\left(-\frac{1}{2}\right), z+\frac{1}{2}, 0\right)
$$

for all $t \geqslant \zeta^{-1}\left(-\frac{1}{2}\right)$.

This implies Property (ii).

We may now perform the iterating process. Some notations first: for any $(t, z)$ in the positivity domain of $p$ we will set, as in [9]:

$$
\hat{\nabla} p(t, z, 0):=\left(\partial_{t} p(t, z, 0), \partial_{z} p(t, z, 0)\right) .
$$

For two vectors $v_{1}$ and $v_{2}$ of $\mathbb{R}^{2}$, we denote by $a\left(v_{1}, v_{2}\right)$ the angle between $v_{1}$ and $v_{2}$. For any $v_{0} \in \mathbb{R}^{2}$ and $\theta \in\left[0, \frac{\pi}{2}\right]$, we denote by $\Lambda\left(v_{0}, \theta\right)$ the cone

$$
\Lambda(v, \theta)=\left\{v \in \mathbb{R}^{2}:\left|a\left(v_{0}, v\right)\right| \leqslant \theta\right\} .
$$

We finally denote by $R_{0}$ the rectangle $R_{0}(\rho)$, where $\rho$ is any number chosen so that Proposition 5.1 works, and by $R_{n}$ the rectangle

$$
R_{n}=\left\{\zeta^{-1}\left(-\frac{1}{4^{n}}\right) \leqslant t \leqslant \zeta^{-1}\left(\frac{1}{4^{n}}\right), \frac{1+\rho}{4^{n}} \leqslant z \leqslant \frac{1}{4^{n}}\right\} .
$$


Set

$$
\left(t_{n}, z_{n}\right)=\left(\zeta^{-1}\left(-\frac{1}{4^{n}}\right),-\frac{1+\rho}{4^{n}}\right) .
$$

Assume that, at the $n$th iteration, we have found a unit vector $v_{n}$ and $\left.\theta_{n} \in\right] 0, \frac{\pi}{2}[\operatorname{such}$ that

$$
\forall v \in \Lambda\left(v_{n}, \theta_{n}\right), \forall(t, z) \in R_{n}, \quad \partial_{\nu} p(t, z) \geqslant 0 .
$$

Let us rescale and consider the function

$$
p_{n}(t, z, x)=4^{n} p\left(\frac{t}{4^{n}}, \frac{z}{4^{n}}, \frac{x}{2^{n}}\right),
$$

the rescaled interface

$$
\zeta_{n}(t)=4^{n} \zeta\left(\frac{t}{4^{n}}\right)
$$

and the rescaled time and space

$$
t_{0}=4^{n} \zeta^{-1}\left(-\frac{1}{4^{n}}\right), \quad z_{0}=-1-\rho .
$$

The rescaled function $p_{n}$ is defined in the rectangle $R_{0}$.

We first note that

$$
\text { (i) } \begin{aligned}
\left|a\left(v_{n}, \hat{\nabla} p_{n}\left(t_{0}, z_{0}, 0\right)\right)\right| \geqslant \theta_{n}, & \\
\text { (ii) } \forall v \in\left[0, \theta_{n}\right], \partial_{\nu} p_{n}\left(t_{0}, z_{0}, 0\right) & =\hat{\nabla} p_{n}\left(t_{0}, z_{0}, 0\right) . v \\
& \geqslant \gamma \cos a\left(v, \hat{\nabla} p_{n}\left(t_{0}, z_{0}, 0\right)\right) .
\end{aligned}
$$

Moreover, we know by assumption that $\partial_{\nu} p \geqslant 0$ in $R_{0}$. Hence the Harnack inequalities, Theorem 4.2, apply and yield the existence of $q>0$ such that

$$
\begin{aligned}
& \forall v \in \Lambda\left(v_{n}, \theta_{n}\right), \forall(t, z) \in\left[\zeta_{n}^{-1}(-1), \zeta_{n}^{-1}(1)\right] \times\left[-1+\rho,-1-\frac{\rho}{2}\right], \\
& \quad \partial_{\nu} p_{n}(t, z, 0) \geqslant q \cos a\left(v, \hat{\nabla} p_{n}\left(t_{0}, z_{0}, 0\right)\right) .
\end{aligned}
$$

Hence for $\varepsilon>0$ small, we have - for some possibly different $q>0$ :

$$
\begin{aligned}
& \forall v \in \Lambda\left(v_{n}, \theta_{n}\right), \forall t \in\left[\zeta_{n}^{-1}(-1), \zeta_{n}^{-1}(1)\right], \\
& \quad \frac{u((t,-1-\rho)+2 \varepsilon v ; 0)}{u(t,-1-\rho, 0)} \geqslant\left(1+2 q \cos a\left(v, \hat{\nabla} p_{n}\left(t_{0}, z_{0}, 0\right)\right)\right) \varepsilon .
\end{aligned}
$$

Let us apply Proposition 5.1(ii) with

$$
\tilde{\varepsilon}:=q \cos a\left(v, \hat{\nabla} p_{n}\left(t_{0}, z_{0}, 0\right)\right)
$$

and

$$
\tilde{u}(t, z, x):=u((t, z)+2 \varepsilon v ; x) .
$$

We obtain, in particular 


$$
\forall(t, z) \in R_{1}, \quad \tilde{u}(t, z, 0) \geqslant(1+\tilde{\varepsilon}) u(t+\tilde{\varepsilon} \lambda z, z, 0) .
$$

Multiplying by $u$, expanding with respect to $\varepsilon$ and letting $\varepsilon$ to 0 yields

$$
\begin{aligned}
& \forall v \in \Lambda\left(v_{n}, \theta_{n}\right), \forall t \in\left[\zeta_{n}^{-1}\left(-\frac{1}{4}\right), \zeta_{n}^{-1}\left(\frac{1}{4}\right)\right], \forall z \in\left[-\frac{1+\rho}{4}, \zeta_{n}(t)[,\right. \\
& \quad \partial_{\nu} p(t, z, 0) \geqslant q \lambda \gamma \cos a\left(v, \hat{\nabla} p_{n}\left(t_{0}, z_{0}, 0\right)\right) .
\end{aligned}
$$

Now, to enlarge the cone $\Lambda\left(v_{n}, \theta_{n}\right)$ we could just rely on the geometrical lemma of [6]; the situation being here much simpler, we give a complete proof of this fact.

By construction we have

$$
\left|a\left(v_{n}, \hat{\nabla} p_{n}\left(t_{0}, z_{0}, 0\right)\right)\right| \leqslant \theta_{n}
$$

assume this angle to be $>0$ : the opposite case is treated in the same fashion. Let us see how far away from the cone $\Lambda\left(v_{n}, \theta_{n}\right)$ we are allowed to pick $v$ without violating the inequality $\partial_{\nu} p(t, z, 0) \geqslant 0$. To simplify the notations, identify $\mathbb{R}^{2}$ with the complex plane. Set

$$
\tilde{v}_{n}=v_{n} \mathrm{e}^{\mathrm{i} \theta_{n}}
$$

We have, for all $(t, z) \in R_{1}$ :

$$
\begin{aligned}
\partial_{\nu} p(t, z, 0) & =|\hat{\nabla} p(t, z, 0)| \cos a(\hat{\nabla} p(t, z, 0), v) \\
& =|\hat{\nabla} p(t, z, 0)| \cos \left(a\left(\hat{\nabla} p(t, z, 0), \tilde{v}_{n}\right)+a\left(\tilde{v}_{n}, v\right)\right) \\
& =\cos a\left(\tilde{v}_{n}, v\right) \partial_{\tilde{v}_{n}}-|\hat{\nabla} p(t, z, 0)| \sin a\left(\hat{\nabla} p(t, z, 0), \tilde{v}_{n}\right) \sin a\left(\tilde{v}_{n}, v\right) \\
& \geqslant q \cos \theta_{n}-|\hat{\nabla} p(t, z, 0)| \sin a\left(\tilde{v}_{n}, v\right)
\end{aligned}
$$

for a possibly different $q$, that can anyway be chosen independent of $n$. Therefore we have $\partial_{\nu} p(t, z, 0) \geqslant 0$ if and only if

$$
\operatorname{tg} a\left(v, \tilde{v}_{n}\right) \leqslant \frac{L}{q \operatorname{tg} \theta_{n}} .
$$

Accordingly, let us set

$$
\begin{aligned}
& \theta_{n+1}=\theta_{n}+\frac{1}{2} \operatorname{Arctg}\left(\frac{L}{q \operatorname{tg} \theta_{n}}\right), \\
& v_{n+1}=v_{n} \mathrm{e}^{\mathrm{i}\left(\theta_{n+1}-\theta_{n}\right) / 2} .
\end{aligned}
$$

We have $\partial_{\nu} p(t, z, 0) \geqslant 0$ in $R_{1}$ for all $v \in \Lambda\left(v_{n+1}, \theta_{n+1}\right)$. Moreover, we may see that the sequence $\left(\theta_{n}\right)_{n}$ converges geometrically to $\frac{\pi}{2}$. Also, scaling back, we have proved the following property:

$$
\forall v \in \Lambda\left(v_{n+1}, \theta_{n+1}\right), \forall(t, z) \in R_{n+1}, \partial_{\nu} p(t, z, 0) \geqslant 0 .
$$

By nondegeneracy, the initiation of the iteration process is trivial. 


\subsection{The $C^{1, \alpha}$ estimate}

Let us summarize the preceding section. There is a sequence of cones $\Lambda\left(v_{n}, \theta_{n}\right)$, with $\theta_{n} \in\left[0, \frac{\pi}{2}\right]$, such that

$$
\begin{aligned}
\text { (i) }\left|v_{n}\right| & =1, \\
\text { (ii) } a\left(v_{n}, v_{n+1}\right) & =\theta_{n+1}-\theta_{n}, \\
\text { (iii) } \theta_{n+1} & \leqslant \theta_{n}+q\left(\frac{\pi}{2}-\theta_{n}\right) \quad \text { with } 0<q<1 .
\end{aligned}
$$

This is the final ingredient to the

Proof of Theorem 1.1. - Properties (ii) and (iii) of Eq. (5.25) imply

$$
\left|\theta_{n+1}-\theta_{n}\right|+\left|v_{n+1}-v_{n}\right| \leqslant C q^{n}
$$

hence there is a unit vector $v_{\infty}$, a Lipschitz function $\zeta_{\infty}(t)$, and a Lipschitz function $p_{\infty}(t, z, x)$ such that the sequences $\left(v_{n}\right)_{n},\left(\zeta_{n}\right)_{n}$ and $\left(p_{n}\right)_{n}$ converge to $v_{\infty}, \zeta_{\infty}$ and $p_{\infty}$ respectivey. The function $p_{\infty}$ is smooth in its positivity set $\{z>\zeta(t)\}$. Moreover we have

$$
\forall v \in\left\{v_{\infty} . v \geqslant 0\right\}, \forall(t, z) \in R_{0}, \quad \partial_{v} p_{\infty}(t, z, 0) \geqslant 0 .
$$

Hence $\zeta_{\infty}(t)$ is a linear function. This implies the differentiability of $\zeta$ at $t=0$; moreover, our analysis being valid at each free boundary point, we end up with the differentiability of $\zeta$ at all $t>0$.

Let now $(t, \zeta(t))$ be another free boundary point, close to $(0,0)$. Let $n$ the unique integer such that

$$
(t, \zeta(t)) \in R_{n} \quad \text { and } \quad(t, \zeta(t)) \notin R_{n+1} .
$$

If $t$ is close enough to 0 , then $n$ is large and, due to the fact that $\dot{\zeta}$ is bounded and bounded away from 0 , we have

$$
\frac{\gamma}{4^{n} L} \leqslant|t| \leqslant \frac{L}{4^{n} \gamma} .
$$

For all $s>0$, let $v(s)$ be the normal vector to the interface $\{z=\zeta\}$; we note that, automatically,

$$
\left|a\left(v_{n}, v(t)\right)\right| \leqslant \frac{\pi}{2}-\theta_{n} .
$$

Consequently there holds

$$
\begin{aligned}
|v(t)-v(0)| & \leqslant\left|v(t)-v_{n}\right|+\left|v_{n}-v(0)\right| \leqslant 2 C q^{n} \\
& \leqslant C|t|^{\alpha}, \quad \text { with } \alpha=-\frac{\log q}{\log 4} .
\end{aligned}
$$

This implies

$$
|\dot{\zeta}(t)-\dot{\zeta}(0)| \leqslant C|t|^{\alpha}
$$

for a possibly different constant $C$; hence the theorem. 


\section{Qualitative properties}

We have now proved that the constructed solution $u$ is a classical one. First, we will show how the strong maximum principle property proved in Sections 4 and 5 will allow us to derive a large time behaviour result. Then, to finish the analogy, we prove the free boundary relation.

\subsection{Large time behaviour}

Let us summarize Propositions 4.5 and 5.1 in the following

Proposition 6.1. - Let $u_{1} \leqslant u_{2}$ two solutions of (1.2), (1.3) such that

$$
\sup _{z \geqslant 0}\left(u_{2}(0, z, 0)-u_{1}(0, z, 0)\right)>0 .
$$

Then we have

$$
\inf _{1 \leqslant t \leqslant 4, z \geqslant 1}\left(u_{2}(t, z, 0)-u_{1}(t, z, 0)\right)>0 .
$$

The proof is omitted.

Proof of Theorem 1.2. - Let $\mathcal{S}(t)$ denote the semigroup generated by the Cauchy problem for (1.2), (1.3) and, for $z_{0} \in \mathbb{R}$, let $\tau_{z_{0}}$ be the translation operator

$$
\tau_{z_{0}} u(t, z, x)=u\left(t, z+z_{0}, x\right) .
$$

Set $u(t):=\mathcal{S}(t) u_{0}$ and

$$
u_{n}(t, z, x)=u\left(4^{n} t, 2^{n} z, 2^{n} x\right), \quad 0 \leqslant t \leqslant 4 .
$$

Let us define the discrete semigroup

$$
\mathcal{T} u_{0}(z, x)=\mathcal{S}(4) u_{0}(2 z, 2 x) .
$$

The theorem will be proved as soon as we have shown the convergence of the sequence $\left(u_{n}\right)_{n}:=\mathcal{T}^{n} u_{0}$ towards a translate of $\phi$. Let $\omega\left(u_{0}\right)$ denote the $\omega$-limit set of $u_{0}$ in $C\left(\mathbb{R}_{+} \times \mathbb{R}_{+}\right)$with respect to $\mathcal{T}$. We first observe that, because there is a Lipschitz bound for $p$, the sequence $\left(u_{n}\right)_{n}$ is relatively compact in $C\left([0,4] \times \mathbb{R}_{+} \times \mathbb{R}_{+}\right)$; hence $\omega\left(u_{0}\right)$ is nonvoid.

By homogeneity, the function $u_{n}$ is a solution of (1.2), (1.3) and there are two bounds of the form

$$
\tau_{z_{0}} \phi \leqslant u_{n} \leqslant \tau_{z_{1}} \phi
$$

independently of $n$. For $\psi \in \omega\left(u_{0}\right)$ consider

$$
h(\psi)=\inf \left\{\hat{z}: \forall z \leqslant \hat{z}, \tau_{z} \phi \leqslant \psi\right\}
$$




$$
h_{0}=\min _{\psi \in \omega\left(u_{0}\right)} h(\psi)
$$

Such an $h_{0}$ is attained by some element $\psi_{0}$ of $\omega\left(u_{0}\right)$. Assume that we do not have $\psi=\tau_{h_{0}} \phi$; then by Proposition 6.1 we have $h(\mathcal{T} \psi)<h_{0}$, contradicting (6.30).

\subsection{The free boundary relation}

We come back to the sequence $\left(p_{n}\right)_{n}$ defined by (5.14) and to its limiting function $p_{\infty}$. Notice that this function $p_{\infty}$ is defined on $(t, z, x) \in \mathbb{R} \times \mathbb{R} \times \mathbb{R}_{+}$.

Proof of Theorem 1.3. - From Eq. (5.27) we infer the fact that, for every $(t, z)$ the vector $\hat{\nabla} p_{\infty}(t, z, 0)$ is proportional to the vector $v_{\infty}$. Therefore let us set $v_{\infty}=\left(v_{1}, v_{2}\right)$; there exists a $C^{1}$ real function $\alpha(t, z)$ such that

$$
\left(\partial_{t} p_{\infty}(t, z, 0), \partial_{z} p_{\infty}(t, z, 0)\right)=\alpha(t, z)\left(v_{1}, v_{2}\right)
$$

We have therefore

$$
v_{1} \alpha_{z}=v_{2} \alpha_{t}
$$

implying that $\alpha(t, z)$ has the form $\alpha_{0}\left(t-\frac{v_{1}}{v_{2}} z\right)$. Hence $p_{\infty}$ is a travelling wave, and hence has a unique form due to Proposition 3.3. In particular, it satisfies the free boundary relation.

\section{Acknowledgements}

The second author gratefulley acknowledges several invitations at the Mathematics Department of the University of Texas at Austin.

\section{REFERENCES}

[1] Aronson D.G., Bénilan P., Régularité des solutions de l'équation des milieux poreux dans $\mathbb{R}^{N}$, C. R. Acad. Sci. Paris 288 (1979) 103-105.

[2] Aronson D.G., Caffarelli L.A., Kamin S., How an initially stationary interface begins to move in porous medium flow, SIAM J. Math. Anal. 14 (1983) 639-658.

[3] Atkinson F.V., Peletier L.A., Similarity profiles of flows through porous media, Arch. Rational Mech. Anal. 42 (1971) 369-379.

[4] Atkinson F.V., Peletier L.A., Similarity solutions of the nonlinear diffusion equation, Arch. Rational Mech. Anal. 54 (1974) 373-392.

[5] Audounet J., Giovangigli V., Roquejoffre J.-M., A threshold phenomenon arising in the propagation of a spherical flame, Physica D 121 (1998) 295-316.

[6] Caffarelli L.A., A Harnack inequality approach to the regularity of free boundaries. I. Lipschitz free boundaries are $C^{1, \alpha}$, Rev. Mat. Iberoamericana 3 (1987) 39-62.

[7] Caffarelli L.A., Vazquez J.-L., Viscosity solutions for the porous medium equation, in: Differential Equations: La Pietra 1996 (Florence), Proc. Sympos. Pure Math., Vol. 65, American Mathematical Society, Providence, RI, 1999.

[8] Caffarelli L.A., Vazquez J.-L., Wolanski N.I., Lipschitz continuity of solutions and interfaces in the $N$-dimensional porous medium equation, Indiana Univ. Math. J. 36 (1987) 373-401. 
[9] Caffarelli L.A., Wolanski N.I., $C^{1, \alpha}$ regularity of the free boundary for the $N$-dimensional porous media equation, Comm. Pure Appl. Math. 43 (1990) 885-902.

[10] Clément P., Gripenberg G., Londen S.-O., Hölder regularity for a linear fractional evolution equation, in: Topics in Nonlinear Analysis, H. Amann Anniversary Volume, Birkhäuser, 1999, pp. 69-82.

[11] Gorenflo R., Vessela S., Abel Integral Equations, Springer, New York, 1991.

[12] Gordeev A.V., Grechikha A.V., Kalda Y.L., Rapid penetration of a magnetic field into a plasma along an electrode, Sov. J. Plasma Phys. 16 (1) (1990) 55-57.

[13] Gripenberg G., Londen S.-O., Fractional derivatives and smoothing in nonlinear conservation laws, Diff. Int. Eq. 8 (1995) 1961-1976.

[14] Henry D., Geometric Theory of Semilinear Parabolic Equations, Springer, New York, 1981.

[15] Ladyzhenskaya O.A., Ural'tceva N.N., Solonnikov V.A., Linear and Quasilinear Equations of Parabolic Type, American Mathematical Society, Providence, RI, 1968.

[16] Méhats F., Thèse de doctorat de l'École polytechnique, 1997.

[17] Méhats F., Roquejoffre J.-M., A nonlinear oblique derivative problem for the heat equation, Part I: Basic results, Ann. Inst. Henri Poincaré, Analyse non linéaire 16 (1999) 221-253.

[18] Méhats F., Roquejoffre J.-M., A nonlinear oblique derivative problem for the Heat equation, Part II: Singular self-similar solutions, Ann. Inst. Henri Poincaré, Analyse non linéaire 16 (1999) 691-724.

[19] Walter W., Differential Inequlities, Springer, Berlin, 1964. 